\title{
Indices de compétition en forêt dense tropicale humide : étude de cas sur le dispositif sylvicole expérimental de Paracou (Guyane française)
}

\author{
Sylvie Gourlet-Fleury*
}

Cirad-Forêt, Programme Forêts Naturelles, BP 701, 97387 Kourou cedex, Guyane française

(Reçu le 9 août 1996 ; accepté le 18 février 1997)

\begin{abstract}
Competition indices in a tropical moist forest: trials on the sylvicultural experimental plots of Paracou (French Guiana). The building of a single tree distance-dependent growth model, using the data collected on the experimental plots of Paracou (French Guiana) led us to review numerous competition indices that have been used in temperate forests since 1950 and to discuss their applicability in moist tropical forests. We kept four simple indices calculated on tree-centered circular plots, as well as their variation in time, and studied their ability to predict diameter growth relative to: sylvicultural treatments, diameter class and species to which the subject tree belongs, circular plot radius used to evaluate the indices. We show that their efficiency remains limited (we could not explain more than $21 \%$ of the observed variance on mean annual diameter growth when all species are taken together): the best results are obtained most often with a one-sided competition index, for small diameter classes, big plot radius (at least $20 \mathrm{~m}$ ) and some light-demanding or canopy species. (C) Inra/Elsevier, Paris.)
\end{abstract}

competition index / diameter growth / neighbourhood / model / moist tropical forest

Résumé - Dans le cadre de la mise au point, sur le dispositif sylvicole de Paracou (Guyane française), d'un modèle de croissance d'arbre dépendant des distances, nous avons passé en revue plusieurs indices de compétition utilisés en forêt tempérée depuis les années 50 et envisagé leur application au cas de la forêt dense tropicale humide. Quatre indices simples calculés sur des placettes circulaires centrées sur l'arbre sujet, ainsi que leur variation dans le temps, ont été retenus et leur efficacité vis-à-vis de la prédiction de l'accroissement en diamètre étudiée en fonction des variables suivantes : type d'intervention sylvicole, classe de diamètre et espèce à laquelle appartiennent les arbres sujets, rayon de la placette sur laquelle sont calculés les indices. Nous montrons que leur intérêt reste limité (toutes espèces confondues, nous n'avons pu « expliquer » plus de $21 \%$ de la variance observée sur l'accroissement moyen annuel en diamètre) ; les meilleurs résultats sont obtenus la plupart du temps avec un indice lié au statut social de l'arbre, pour les petites classes de diamètre, des placettes de grande taille (au moins $20 \mathrm{~m}$ de rayon) et certaines espèces à tempérament héliophile ou structurantes de la voûte forestière. (C) Inra/Elsevier, Paris.)

indice de compétition / accroissement / voisinage / modèle / forêt dense tropicale humide

\footnotetext{
* Correspondance et tirés à part

Campus international de Baillarguet, BP 5035, 34032 Montpellier cedex 1, France
} 


\section{INTRODUCTION}

\subsection{Contexte de I'étude}

L'étude des phénomènes de compétition en forêt dense tropicale humide s'insère dans un cadre plus large de recherche sur les mécanismes qui régissent le fonctionnement de cet écosystème, afin de donner au gestionnaire forestier les moyens d'intervenir plus rationnellement dans les peuplements. Si, en forêt tempérée, il dispose pour cela d'une masse de connaissances et d'outils élaborés [19, 33, 34], en forêt tropicale naturelle beaucoup reste à faire.

En Guyane française, la forêt dense ombrophile sempervirente de plaine couvre environ $90 \%$ des 8,5 millions d'hectares du département, et la gestion de la quasi-totalité de cette superficie est confiée à l'Office National des Forêts (ONF). Seule la partie nord du massif forestier, située le long de la côte sur une profondeur de 60 à 70 kilomètres $(1,5$ millions d'hectares), fait l'objet d'un aménagement forestier global.

La pratique des éclaircies, bien maitrisée en zone tempérée, pose de nombreux problèmes en forêt dense tropicale humide, par exemple :

i) quelles règles simples de sylviculture adopter dans des peuplements aussi hétérogènes?

ii) comment procéder aux éclaircies (abattage, annélation, empoisonnement des arbres que l'on souhaite éliminer au profit des autres)?

iii) quels seront les effets des interventions sur la dynamique des peuplements traités, sur leur composition floristique, sur les populations animales qu'ils abritent et dont ils dépendent, sur la qualité technologique des futurs arbres récoltés, etc. ?
De nombreuses études expérimentales ont été entreprises ou sont en cours pour tenter d'apporter une réponse à ces questions (voir synthèse dans [13]). Parallèlement à l'approche expérimentale, nécessitant d'importants moyens humains et financiers ainsi qu'un certain recul dans le temps, une approche basée sur la modélisation, qui permet de s'affranchir partiellement de l'ensemble de ces contraintes, est mise en œuvre depuis 1992 au Cirad-Forêt dans le domaine de la dynamique des peuplements forestiers, tant au niveau du peuplement [23] qu'au niveau de l'arbre [28]. C'est ce dernier niveau qui nous intéresse ici plus particulièrement.

\subsection{Objectifs}

De nombreux éléments influencent la croissance d'un arbre [3] notamment son patrimoine génétique et son accès aux ressources (lumière, eau ou nutriments) contrôlé en partie par la compétition. L'essentiel des efforts de quantification de cette compétition par des indices adaptés a porté sur les peuplements forestiers tempérés, et plus rarement sur les peuplements naturels tropicaux.

Nous passons d'abord en revue les grandes familles d'indices avant de faire un point rapide sur leur possibilité d'application en forêt dense tropicale humide. Puis nous présentons les conclusions d'une première étude exploratoire réalisée en 1991-1992 sur une parcelle du dispositif de Paracou, visant à identifier des indices pouvant participer à l'élaboration d'un modèle de croissance. Nous prolongeons ce travail en introduisant de nouvelles variables, dont l'efficacité pour la «prédiction» de la croissance des arbres sera testée de manière systématique sur nos données, toutes espèces confondues dans un premier temps, puis pour certaines espèces particulières choisies en fonction de leur tempérament écologique. 


\section{MATÉRIEL ET MÉTHODES}

\subsection{Matériel}

Conçu en 1982, le dispositif sylvicole de Paracou a été installé en 1984 sur la commune de Sinnamary à environ $50 \mathrm{~km}$ au nord-ouest de Kourou [45, 56-58]. Douze parcelles de 9 ha chacune, soit 108 hectares au total, ont été assises dans une zone de forêt intacte, après inventaire et étude pédologique préalables. Neuf d'entre elles ont fait l'objet, entre 1986 et 1988, d'interventions sylvicoles d'intensité variable (trois types de traitement, appliqués chacun à trois parcelles), les trois dernières jouant le rôle de témoin. Les interventions ont été les suivantes :

i) traitement 1 : exploitation forestière de type "traditionnel » pour le bois-d'cuvre. Environ dix tiges par hectare faisant plus de 50 à $60 \mathrm{~cm}$ de diamètre à $1,30 \mathrm{~m}$ et appartenant à une cinquantaine d'espèces différentes ont été prélevées ;

ii) traitement 2 : exploitation forestière pour le bois-d'œuvre suivie d'une éclaircie par dévitalisation éliminant les arbres de plus de $40 \mathrm{~cm}$ de diamètre à $1,30 \mathrm{~m}$, ne présentant pas d'intérêt commercial potentiel ;

iii) traitement 3 : exploitation forestière pour le bois-d'œuvre du même type que les précédentes, suivie d'une exploitation devant fournir du bois combustible (tiges d'espèces non commerciales faisant entre 40 et $50 \mathrm{~cm}$ de diamètre), et d'une éclaircie par dévitalisation (tiges d'espèces non commerciales faisant plus de $50 \mathrm{~cm}$ de diamètre).

Le tableau I présente, pour chaque traitement, l'évolution du nombre d'arbres présents et de la surface terrière correspondante entre 1984 et 1994.

Depuis 1984, le peuplement constitué de toutes les tiges de plus de $10 \mathrm{~cm}$ de diamètre à $1,30 \mathrm{~m}$ situées sur les 6,25 ha centraux de chaque parcelle (soit environ 46000 arbres au total) fait l'objet d'une mesure annuelle des circonférences par le Cirad-Forêt. Tous les arbres sont numérotés, repérés par des coordonnées cartésiennes et la plupart sont identifiés botaniquement.

\subsection{Méthodes}

\subsubsection{Quelques considérations sur les indices de compétition}

De nombreux auteurs ont étudié les différents indices mis au point depuis les années 50 $[8,15,17,26,37,53]$. Rappelons que les relations de «compétition» entre individus ne sont que l'un des nombreux aspects que peuvent revêtir les interactions entre arbres [36]. La compétition se caractérise par "l'apparition d'effets déprimants entraînés par la mise en présence des organismes " (Malcom, 1966, cité par [36]). C'est essentiellement elle qui régule l'accès d'un individu aux ressources qui lui sont nécessaires, d'où l'intérêt d'essayer de quantifier ce phénomène le mieux possible. C'est la fonction que tentent de remplir les indices de compétition, quelle que soit la famille à laquelle ils appartiennent : indices indépendants ou indices dépendants de la distance.

\subsubsection{Indices indépendants de la distance}

Leur calcul ne nécessite aucune connaissance sur la position des arbres du peuplement étudié et ils peuvent être construits avec des données issues d'inventaires classiques de terrain. Ils peuvent être basés sur la dimension des houppiers, traduisant le fait que les arbres ne se développent pas de la même manière en situation isolée et en peuplement : exemple du Crown Competition Factor de Krajicek et al. [41] des indices de Mitchell $[46,47]$ ou du Crown Ratio de Daniels et al. (1975, cité par [14]). Ou bien ils utilisent la surface terrière (ou le diamètre) et la hauteur des arbres voisins, de manière absolue ou relativement aux dimensions de l'arbre sujet : indices de Steneker et Jarvis [61], Basal Area Index de Glover et Hool [25], etc. À noter que dans la plupart des cas, les compétiteurs, c'est-à-dire les voisins intervenant dans le calcul de l'indice, sont situés à l'intérieur d'une placette circulaire de rayon arbitrairement fixé, centrée sur le sujet. Mais l'ensemble du peuplement étudié peut également être pris en compte : Kohyama [38-40] construit ainsi un one-sided competition index, à partir de la surface terrière cumulée de tous les arbres du peuplement de taille supérieure à celle de chaque sujet. 
Tableau I. Effectifs totaux par hectare (arbres faisant plus de $10 \mathrm{~cm}$ de diamètre à $1,30 \mathrm{~m}$ ) et surface terrière correspondante, dans chaque groupe de trois parcelles correspondant aux différents traitements réalisés à Paracou. L'exploitation a eu lieu d'octobre 1986 à mai 1987, et la dévitalisation de décembre 1987 à janvier 1988. D'après Prothery [54].

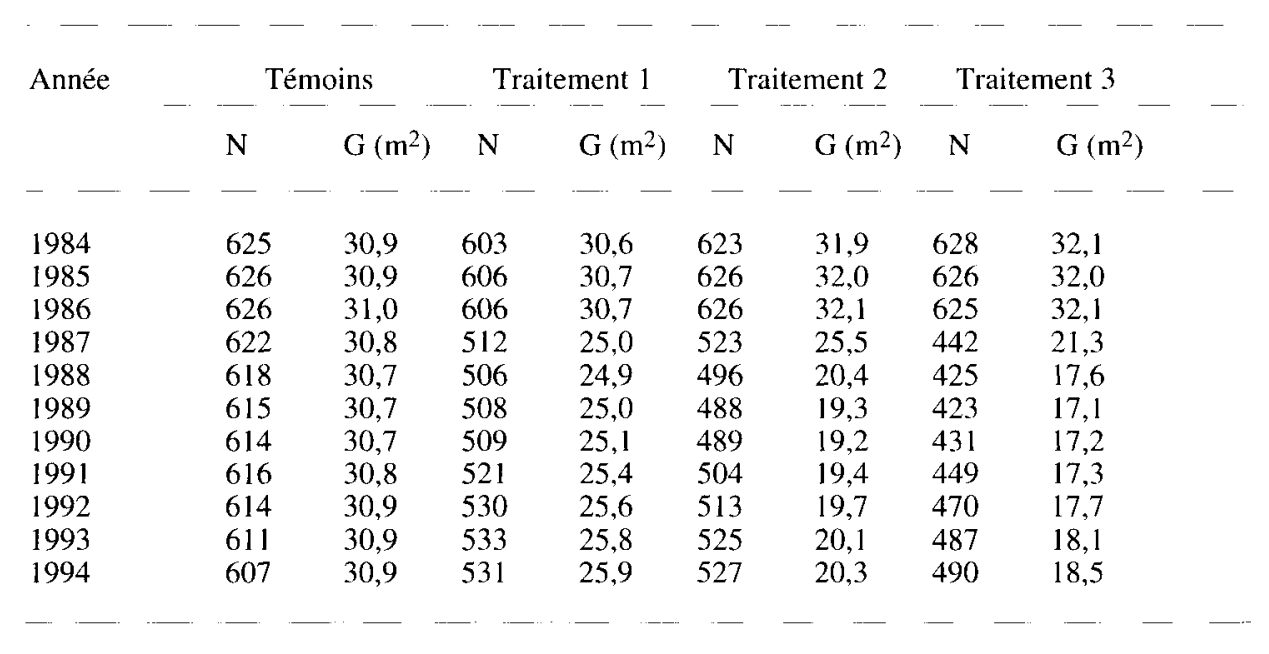

\subsubsection{Indices dépendants de la distance}

Leur calcul fait cette fois intervenir la distance entre arbres, et nécessite de disposer de ces données, ou au moins d'une cartographie des individus. On peut distinguer cinq sousfamilles d'indices de ce type (la nomenclature utilisée pour les trois premières a été empruntée à Daniels et al. [15] :

- Indices de chevauchement (overlap indices)

Le choix des compétiteurs et l'expression de la compétition reposent ici sur le concept de zone d'influence d'un arbre. Celleci est en général circulaire, centrée sur l'arbre et censée représenter la surface maximale d'étendue horizontale qu'il pourrait utiliser pour sa croissance [53]. Son extension est souvent reliée à l'extension maximale du houppier en situation de croissance libre, ou de manière plus ou moins empirique à différentes variables individuelles (diamètre, hauteur). Les compétiteurs sont alors les arbres dont les zones d'influence chevauchent celle du sujet, et l'importance de ces chevauchements est utilisée, de manière additive, pour quantifier la compétition subie par ce dernier.
Une forme générale d'indice fréquemment utilisée est la suivante :

$$
I C_{i}=\frac{b_{1}}{A_{\mathrm{i}}} * \sum_{j=1}^{n c} a_{i j} *\left(\frac{E_{j}}{E_{j}}\right)^{b_{2}}
$$

$i$ et $j$ désignant respectivement l'arbre sujet et ses compétiteurs, nc le nombre de compétiteurs de l'arbre $i, A$ la superficie de la zone d'influence, $a$ la superficie du chevauchement entre les zones d'influence et $E$ un descripteur quelconque de l'arbre tel son diamètre [6]. $b_{1}$ et $b_{2}$ sont des constantes.

De tels indices ont été développés par de nombreux auteurs (Staebler, 1951 et Newnham, 1964 cités par [37], Arney, 1973 cité par Smith et Bell [59], [6, 20, 24]).

- Indices utilisant les ratios taille/distance (size ratio distance indices)

Le principe retenu dans la formulation de ces indices est le suivant : un arbre de gros diamètre éloigné du sujet exerce en théorie une pression de compétition équivalente à celle d'un arbre de petit diamètre, proche du sujet. On peut réunir ces indices sous une formule générale : 


$$
I C_{i}=b_{1} * \sum_{j=1}^{n c} \frac{\mathrm{D}_{j}^{b_{2}}}{\mathrm{D}_{i}^{b_{3}}} * \frac{E_{j}}{L_{i j} b_{4}}
$$

$i$ et $j$ désignant respectivement l'arbre sujet et ses compétiteurs, $n c$ le nombre de compétiteurs de l'arbre $i, D$ le diamètre à $1,30 \mathrm{~m}, L$ la distance entre les arbres et $E$ un descripteur quelconque de l'arbre tel le rayon de sa couronne [30], lorsqu'il ne vaut pas $1 . b_{1}, b_{2}, b_{3}$ et $b_{4}$ sont des constantes.

Les compétiteurs sont désignés de différentes manières : situés dans une placette circulaire de rayon fixé centrée sur le sujet [30, $32,61]$, ou bien désignés selon la méthode du relascope de Bitterlich $[14,60]$.

\section{- Indices de polygones (polygon indices)}

Le concept utilisé ici est proche de celui de la zone d'influence : on fait une nouvelle fois appel à une représentation de l'espace utilisé par un arbre pour se développer (Area Potentially Available de Brown (1965), notamment cité par Moore et al. [48], espace vital de Bouchon [9]). Cet espace vital a la forme d'un polygone entourant le sujet, et les compétiteurs sont les voisins qui participent à la construction du polygone : ses côtés sont perpendiculaires aux segments sujet/voisin, et situés à une distance variable, éventuellement bornée, du dit sujet (tessellation de Dirichlet ou pavage de Voronoï, Bouchon, [10]). La surface du polygone, ou son volume si l'on connaît la hauteur des arbres, sont considérés comme des indicateurs de la pression de compétition subie [2, 15, 35, 48, 49] et Pelz, 1978, cité par [15].

\section{- Autres indices}

Certains indices, plus complexes, sont construits en combinant plusieurs des méthodes évoquées précédemment : exemple du zone count de Opie [52], indice de chevauchement pour lequel la zone d'influence de chaque arbre est déterminée par la méthode du relascope de Bitterlich, ou du PAGAS (proportionally available growing-space area of sectors) de Alemdag [2], faisant également appel au relascope et au système des polygones.

Hamilton [30], Hatch et al [31], Pretzsch [55], entre autres ont construit des indices en
« trois dimensions », calculés à l'aide d'équations de profil de houppiers assimilés à des formes géométriques simples (cônes, sphères, paraboloïdes) : ils expriment soit la compétitivité des sujets, soit la pression de compétition qu'ils subissent.

Il existe enfin des indices particuliers difficiles à rattacher aux familles précédentes, tels que l'estimateur de Latham [42] qui utilise des couples stéréoscopiques de photographies aériennes, ou les indices calculés à l'aide de variogrammes [5].

\subsubsection{Efficacité des indices de compétition dans la prédiction de la croissance}

Incorporés dans les modèles de dynamique forestière, les indices de compétition interviennent pour moduler la croissance potentielle des arbres et la mortalité. Leur efficacité, telle que rapportée par différents auteurs, est extrêmement variable et dépend notamment de la nature de la variable croissance expliquée (accroissement en diamètre, en surface terrière, en hauteur, en volume, accroissement annuel ou sur plusieurs années) et du type de peuplement sur lequel ils sont testés (équienne monospécifique, inéquienne plurispécifique, jeune, âgé, dense, peu dense, etc.).

La plupart des auteurs travaillant sur des peuplements équiennes s'accorde à reconnaître que le meilleur prédicteur de l'accroissement, en diamètre ou en surface terrière, est le diamètre initial qui « explique » (au sens statistique) 30 à $80 \%$ de sa variabilité. Dans ce type de peuplement, le diamètre peut en effet être considéré comme une bonne mesure du statut social de l'arbre. L'adjonction d'un indice de compétition n'améliore pas énormément la précision du modèle, le plus souvent parce qu'il est, par construction, corrélé au diamètre initial du sujet [26].

Les indices généralement reconnus comme les plus efficaces appartiennent aux sousfamilles des indices de chevauchement et de polygones, traduisant l'importance de la notion de «zone d'influence » ou d'« espace vital » autour d'un arbre et ce dans tous les types de peuplement (équiennes monospécifiques à inéquiennes plurispécifiques). Par ailleurs, la prise en compte de la taille respective des individus en présence est très importante (intervention du statut social, qui peut 
être approché de manière qualitative - voir en particulier les travaux de Tomé et Burkhart [65] - ou quantitative, à partir des données de hauteur).

\subsubsection{Applicabilité à la forêt dense tropicale humide}

La grande majorité des études réalisées sur les indices de compétition concerne les forêts tempérées. Des milieux relativement simples, au regard de l'hétérogénéité rencontrée en forêt dense tropicale humide : diversité floristique (de 50 à 250 espèces arborescentes à l'hectare - voir Swaine et al. [63], pour une comparaison de différents sites-) et hétérogénéité structurelle notamment. Quelques travaux testant et utilisant des indices de compétition en forêt tropicale peuvent cependant être mentionnés : Dawkins [16] dans différentes forêts denses d'Afrique, d'Amérique latine et d'Asie) et Wadsworth et al. [68] (en forêt dense de Puerto Rico) ont travaillé essentiellement sur la dimension des sujets (diamètre initial, hauteur, largeur et intensité d'éclairement des houppiers) ou sur la densité globale ou locale des peuplements, dans leur recherche de prédicteurs de la croissance. Besse [7] a testé quelques indices indépendants de la distance en Côte d'Ivoire. Wan Razali [67] a utilisé des indices de densité globale (calculés sur des placettes de 0,4 ha) dans les forêts mixtes de Malaisie et a inspiré les travaux de Chai et Lemay [12] sur les forêts marécageuses de ce pays. Toujours en Malaisie (partie occidentale de Sumatra), Kohyama [38] a testé un indice global traduisant le statut social des individus. Favrichon [21] a travaillé sur la liaison intensité d'éclairement des houppiers (codes de Dawkins) / croissance d'une espèce de forêt centrafricaine. Vanclay [66] a eu recours à des indices de densité globale (placettes de 0,04 à 0,5 ha) dans l'étude des forêts du Queensland.

Dans ce type de milieu, les potentialités de croissance d'un individu vont a priori fortement dépendre de variables telles que :

i) le type d'espèce à laquelle il appartient : hard gambler [51], ou " espèce héliophile des strates intermédiaires et supérieures " [43], caractérisée par des diamètres moyens, une durée de vie courte et une croissance très rapide, à hard struggler, ou « espèce de sousbois ou des strates intermédiaires, tolérante à l'ombrage », caractérisée elle par des diamètres petits à moyens, de longues durées de vie et des croissances lentes, avec tous les intermédiaires entre ces deux extrêmes ;

ii) son stade architectural $[18,29,50]$ : appartenance à l'ensemble du passé, du présent, ou de l'avenir, traduisant en particulier son aptitude à réagir en croissance aux ouvertures du couvert ;

iii) son statut social : en forêt tropicale, Dawkins (cité par [64]) a mis au point une classification largement utilisée, mélangeant statut social et mode d'éclairement des houppiers. Il a obtenu des coefficients de corrélation linéaire allant de 0,40 à 0,47 entre ses codes et l'accroissement en diamètre des individus de certaines espèces de forêt dense africaine ; des résultats similaires ont été obtenus par [21] en République Centrafricaine.

Un certain nombre d'indices de compétition construits en pays tempéré sont difficilement transposables à ce type de forêt :

i) soit pour des raisons techniques : certaines variables telles que hauteur totale des arbres ou dimensions des houppiers sont difficiles à mesurer, du fait d'une visibilité le plus souvent réduite ;

ii) soit pour des raisons fonctionnelles : assimiler les houppiers à des sphères ou des cônes, par exemple, risque d'être trop simplificateur dans des forêts où la quête de la lumière leur fait subir des déformations très importantes ; par ailleurs, les relations de commensalisme semblent suffisamment fréquentes pour rendre peu intéressants les indices trop sensibles à la distance entre arbres, de type size ratio distance [27].

On peut enfin noter que contrairement à ce que l'on observe dans les peuplements équiennes, le diamètre initial des arbres dans ce type de forêt ne constitue généralement pas un très bon indicateur de leur croissance ultérieure. Alder [1] signale, pour différentes espèces de forêts tropicales humides des différents continents, que cette variable ne prend pas en compte plus de 20 à $40 \%$ de la variance observée sur les accroissements. Nous reviendrons plus loin sur ce résultat. 


\subsubsection{Application au dispositif sylvicole de Paracou}

Seules huit parcelles du dispositif (soit deux par traitement) ont été utilisées dans notre étude, qui a porté sur les arbres présents et vivants de 1985 à 1991 , ne présentant aucune anomalie dans les mesures et situés à une distance suffisante des bords des parcelles pour que des indices puissent être calculés sur des placettes circulaires de différent rayon. La prise en compte de l'ensemble des traitements nous a permis de disposer de types d'environnement très contrastés, plutôt fermés en parcelles témoins et parcelles exploitées et ouverts à très ouverts dans les parcelles éclaircies.

NB. Un récapitulatif des notations utilisées dans le texte qui suit figure en annexe.

\subsubsection{Choix de la variable croissance à prédire}

Nous avons choisi de travailler sur l'accroissement en diamètre des arbres, lissé sur trois années consécutives de manière à limiter l'influence des erreurs de mesure. Nous avons pour cela ajusté systématiquement, pour chaque arbre $i$, le modèle suivant :

$$
D_{i, t}=a_{i} t+b_{i}+u_{i, t}
$$

( $D_{i, t}$ diamètre en $\mathrm{cm}$ de l'arbre $i$ au temps $t$, $a_{i}$ et $b_{i}$ : paramètres à estimer, $u_{i}$ écart entre valeur prédite et observée) aux quatre mesures prises en 1988, 1989, 1990 et 1991, soit après traitement sylvicole. $\Delta D_{i}=a_{i}$ représente l'accroissement moyen annuel en diamètre de l'arbre i, c'est la variable "dépendante » que nous voulons prédire à l'aide des indices de compétition.

\subsubsection{Choix des indices de compétition}

Les données réunies sur le dispositif rendent possible l'étude de nombreux indices, à l'exclusion de ceux qui sont basés sur des mesures de hauteur et de dimensions des houppiers. Une collecte de données supplémentaires réalisée sur une des parcelles en 1992 [27] a dans un premier temps permis de comparer :

- i) cinq indices indépendants de la distance : statut social, codes de Dawkins, nombre de voisins « gênants » (gêne appréciée subjectivement), nombre total de voisins de plus de $10 \mathrm{~cm}$ de diamètre (NBT) et surface terrière cumulée (STT) sur une placette circulaire de rayon $\mathrm{R}$ variant de 5 à $10 \mathrm{~m}$, centrée sur l'arbre dont on étudiait l'accroissement en diamètre ;

- ii) et quatre indices dépendants de la distance (indices de Steneker et Jarvis [61]), utilisant des ratios taille/distance.

Ce premier travail a montré :

- i) l'importance pour la croissance, toutes espèces confondues, de l'accès des houppiers à la lumière, la classification de Dawkins se révélant être la meilleure variable prédictrice (résultats conformes à ceux obtenus par [19, 21]);

- ii) l'inadaptation de certains indices utilisant des ratios taille/distance, ce qui s'explique sans doute en partie par l'existence de relations de commensalisme entre arbres poussant très proches les uns des autres (l'un des individus tire profit de la présence de l'autre, alors que sa proximité devrait représenter une gêne importante) ;

- iii) l'efficacité comparable d'indices simples du type NBT et STT et d'indices plus compliqués du type de ceux de Steneker et Jarvis ;

- iv) l'importance de l'effet « espèce ». Les différences de comportement qui les caractérisent se traduisent dans la structuration verticale de la végétation : certaines espèces demeurent en sous-étage tandis que d'autres atteignent la voûte forestière. Cela influe fortement sur des variables telles que « statut social ", " codes de Dawkins " et «nombre de voisins gênants ». Par ailleurs, la sensibilité à la compétition varie vraisemblablement d'un type comportemental à l'autre et le même indice de compétition ne convient pas à toutes les espèces.

Nous avons voulu reprendre la recherche d'indices « efficaces " en utilisant cette fois des parcelles ayant subi tous les types de traitement sylvicole, ce qui permettait de trouver des arbres dans toutes les situations de compétition possibles. Nous avons retenu les indices les plus simples déjà étudiés exprimant une densité locale (NBT et STT) et décidé d'en rajouter d'autres prenant en compte de manière indirecte les problèmes de statut 
social dont l'importance a été soulignée plus haut, toujours sur des placettes de rayon $\mathrm{R}$, à savoir :

- i) le nombre de tiges vivantes, de taille supérieure ou égale à celle du sujet (NBD) ; (STD).

- ii) la surface terrière correspondante

Ces derniers indices nous ont permis de nous affranchir du problème de l'attribution aux arbres de codes de Dawkins, information non disponible sur l'ensemble du dispositif.

Nous avons décidé par ailleurs de pallier le problème de l'aspect statique inhérent aux indices de compétition (critiqué notamment par [11]) en nous intéressant à leur évolution passée. On sait en effet que la mortalité, par chablis ou sur pied, constitue le moteur principal de la dynamique des peuplements. De manière logique, lorsqu'un arbre disparaît, il libère de l'espace et des ressources pour ses voisins. A priori un arbre autour duquel la pression de l'environnement diminue (disparition d'arbres, entraînant une baisse de la valeur des indices de compétition), doit voir sa croissance stimulée. À l'inverse, il doit se trouver gêné en cas d'augmentation de la pression (recrutement, augmentation importante de la croissance des voisins, entrainant une augmentation de la valeur des indices). La prise en compte de cet historique a été faite au travers du calcul de l'évolution, entre 1985 et 1988 , des quatre indices statiques retenus (valeur calculée en 1988), pour obtenir quatre indices «dynamiques »: DNBT, DSTT, DNBD et DSTD. La période $1985 / 1988$ étant celle durant laquelle ont été effectuées les interventions sylvicoles, était également celle qui permettait d'obtenir la plus large gamme de valeurs des indices intéressés ${ }^{1}$.

Tableau II. Valeur moyenne en 1988 des principaux indices de compétition étudiés sur huit parcelles de Paracou, pour des placettes de rayon $30 \mathrm{~m}$ (10 745 individus). Toutes espèces confondues.

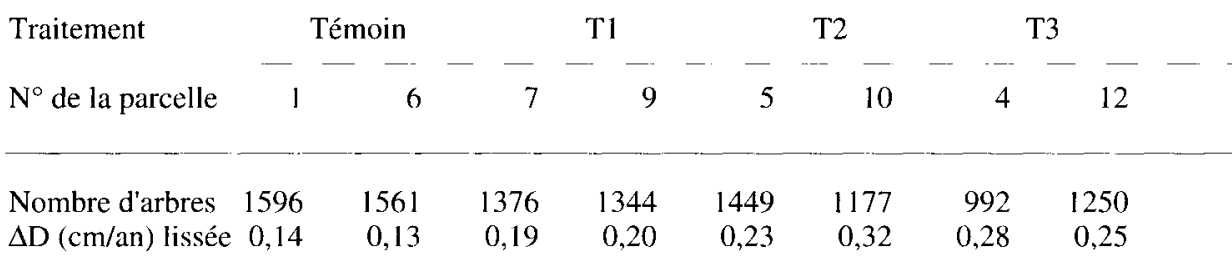

Valeur moyenne des indices sur la parcelle

\begin{tabular}{|c|c|c|c|c|c|c|c|c|}
\hline NBT & 168,1 & 159,0 & 149,2 & 147,6 & 161,3 & 138,4 & 128,0 & 149,0 \\
\hline DNBT & $-4,9$ & $-1,9$ & $-28,2$ & $-28,8$ & $-28,7$ & $-34,2$ & $-59,0$ & $-40,7$ \\
\hline $\operatorname{STT}\left(\mathrm{m}^{2}\right)$ & 8,3 & 8,3 & 7,7 & 7,0 & 6,2 & 5,9 & 5,2 & 5,9 \\
\hline $\operatorname{DSTT}\left(\mathrm{m}^{2}\right)$ & $-0,2$ & 0,0 & $-1,4$ & $-1,4$ & $-2,4$ & $-3,3$ & $-3,8$ & $-3,2$ \\
\hline NBD & 89,0 & 81,8 & 76,3 & 74,9 & 81,8 & 70,6 & 65,5 & 75,4 \\
\hline DNBD & $-4,5$ & $-2,8$ & $-14,2$ & $-14,7$ & $-19,0$ & $-21,7$ & $-33,3$ & $-23,8$ \\
\hline $\operatorname{STD}\left(\mathrm{m}^{2}\right)$ & 6,7 & 6,7 & 6,0 & 5,4 & 4,7 & 4,5 & 3,9 & 4,4 \\
\hline $\operatorname{DSTD}\left(\mathrm{m}^{2}\right)$ & $-0,3$ & 0,0 & $-1,2$ & $-1,2$ & $-2,3$ & $-3,1$ & $-3,4$ & $-3,0$ \\
\hline -. & - & -- & - & $\cdots$ & - & ... & - & - \\
\hline
\end{tabular}

N.B. DNBT, DSTT, DNBD et DSTD représentent la variation de l'indice correspondant (resp NBT, STT, NBD et STD) entre 1985 et 1988.

1 Donc, l'accroissement moyen annuel en diamètre est calculé entre 1998 et 1991, tandis que NBT, STT, NBD et STD sont calculés en 1988 soit en début de période de croissance et DNBT, DSTD, DNBD et DSTD sont calculés entre 1985 et 1988 (on respecte un pas de trois ans). 
Un des problèmes pratiques posés par ces indices consistait en la détermination du rayon $R$ des placettes permettant didentifier les voisins intervenant dans le calcul. En théorie, ce rayon devrait correspondre à la distance maximale prospectée par l'arbre pour s'approvisionner en lumière, en eau et en éléments minéraux : on retrouve ici la notion d'« espace vital » évoquée plus haut. Nous avons donc systématiquement étudié des rayons allant de 10 à $40 \mathrm{~m}$ par pas de $5 \mathrm{~m}$.

Le tableau $/ /$ reprend la valeur moyenne de tous les indices étudiés sur les différentes parcelles retenues, pour un rayon de placette de $30 \mathrm{~m}$. Il permet notamment de se faire une idée de l'impact des traitements sylvicoles. Par ailleurs, la distribution de quelques-uns d'entre eux est représentée figure 1 .

\subsubsection{Mesures de l'efficacité des indices}

Nous avons recherché les indices les plus liés à l'accroissement étudié, en passant par le calcul de coefficients de corrélation linéaire simples et l'étude des résultats de régressions linéaires multiples. Parmi les problèmes qui se sont posés :

- i) l'hétéroscédasticité des résidus des régressions de type $\Delta \mathrm{D}=\mathrm{f}(\mathrm{IC}, \mathrm{DIC})(\mathrm{IC}=$ indice de compétition), qui nous a conduit à travailler non pas sur la variable $\Delta \mathrm{D}$, mais sur sa transformée logarithmique $\log (\Delta \mathrm{D}+\mathrm{c})(\mathrm{c}$ : constante positive permettant de traiter les faiblesaccroissements négatifs fréquents dans ce type de forêt. Voir figure 2);

- ii) la non-indépendance des individus étudiés en croissance : en toute rigueur, il n'aurait fallu travailler que sur des arbres présents sur des placettes non chevauchantes, mais cela aurait considérablement réduit le jeu de données : ainsi, pour des placettes de rayon $30 \mathrm{~m}$, seuls 48 arbres auraient pu être retenus, ce qui interdisait notamment toute étude par classe de diamètre ou par espèce.
Nous avons calculé systématiquement, dans toutes les études de corrélation linéaire, le coefficient de rang de Spearman en plus du coefficient classique de Pearson. Les valeurs obtenues étant cependant très proches, et les conclusions en terme de significativité toujours concordantes, nous n'avons fait figurer sa valeur que dans le tableau III.

Chaque étude de corrélation linéaire entre accroissement et indices, que ce soit en fonction des traitements sylvicoles, des classes de diamètre ou des espèces, a été complétée par la recherche du meilleur modèle linéaire à deux variables (lorsque la prise en compte d'un deuxième indice avait un sens) $\log (\Delta \mathrm{D}+\mathrm{c})=\mathrm{f}(\mathrm{IC} 1, \mathrm{IC} 2)$, en faisant appel à des régressions pas à pas maximisant le coefficient de détermination ${ }^{2}$. Ce dernier, ainsi que l'identité de IC1 et IC2 figurent dans tous les tableaux concernés.

La recherche d'un rayon « optimal » de placette a été réalisée de deux manières :

- i) en comparant les coefficients de corrélation simple ou multiple accroissement / indices de compétition sur des placettes de taille croissante ;

- ii) en recalculant les indices par tranches successives de $10 \mathrm{~m}$, jusqu'à une distance de $50 \mathrm{~m}$ des sujets, selon le schéma de la figure $3 a$ et en vérifiant que l'apport d'informations réalisé par chaque tranche successive possédait un intérêt réel par rapport à celui d'une placette initiale de taille $10 \mathrm{~m}$. Nous avons pour cela comparé des modèles emboîtés selon le principe suivant : nous sommes partis du meilleur modèle linéaire à deux variables $\log (\Delta D+c)=f(I C I, I C 2)$ ajusté pour des placettes de rayon $10 \mathrm{~m}$, puis nous avons inclus à ce modèle les variables IC1 et IC2 calculées pour la «tranche de distance » $10 \mathrm{~m}-20 \mathrm{~m}$, en vérifiant au travers d'un test $F$ l'utilité de cette inclusion. Et ainsi de suite jusqu'à $50 \mathrm{~m}$.

2 Les indices de compétition sélectionnés à l'issue de cette étude, censés traduire l'influnce de l'environnement, devaient intervenir par la suite dans la formulation d'un modèle de croissance plus complet. Nous avons donc délibérément limité la sélection à deux indices, de manière à ne pas alourdir le modèle finnal et conserver des expressions aisément manipulables et interprétables. Il faut noter par ailleurs que l'ajustement de ces modèles avait un but essentiellement exploratoire et que les hypothèses d'homoscédasticité et de normalité des résidus n'ont pas été systématiquement vérifiées : les résultats obtenus ont une valeur essentiellement indicative. 


\section{(a)}

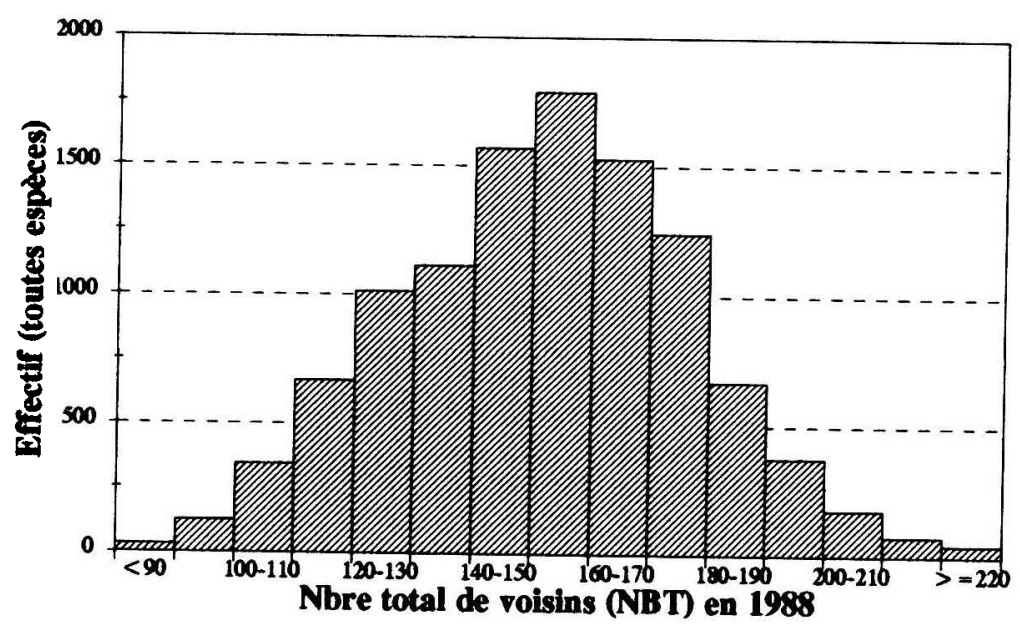

(b)

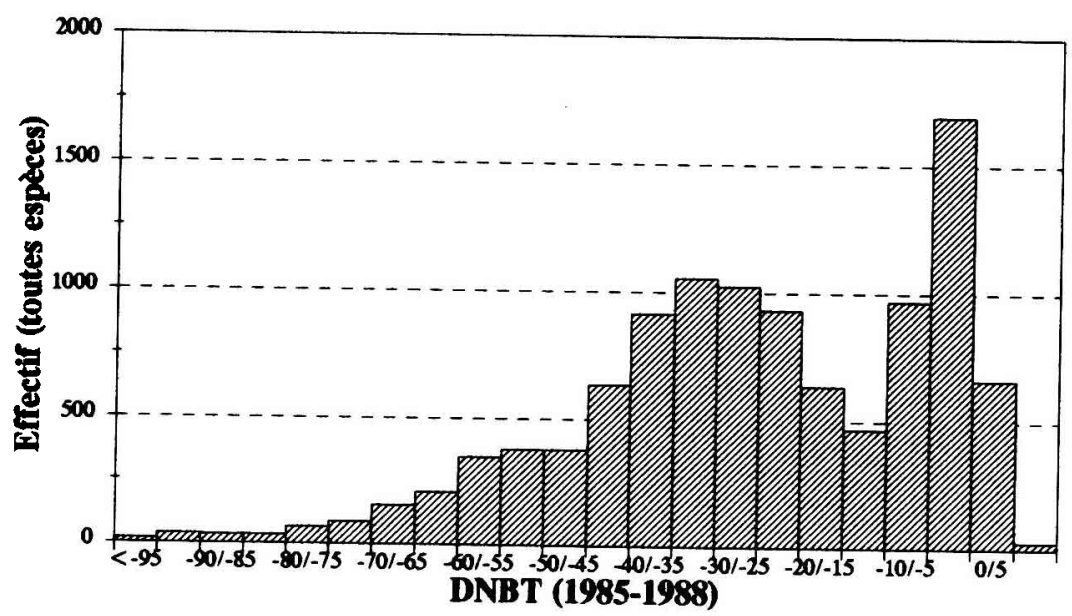

Figure 1. Distribution de quelques indices de compétition calculés sur des placettes de rayon 30 $\mathrm{m}$ : (a) nombre total de voisins en 1988 ; (b) évolution de ce nombre entre 1985 et 1988 ; (c) nombre de voisins de diamètre supérieur à celui du sujet en 1988 ; (d) évolution de ce nombre entre 1985 et 1988. (e) Distribution des arbres sujets en classes de diamètre, en 1988. NB. 10745 individus sont pris en compte. 
(c)

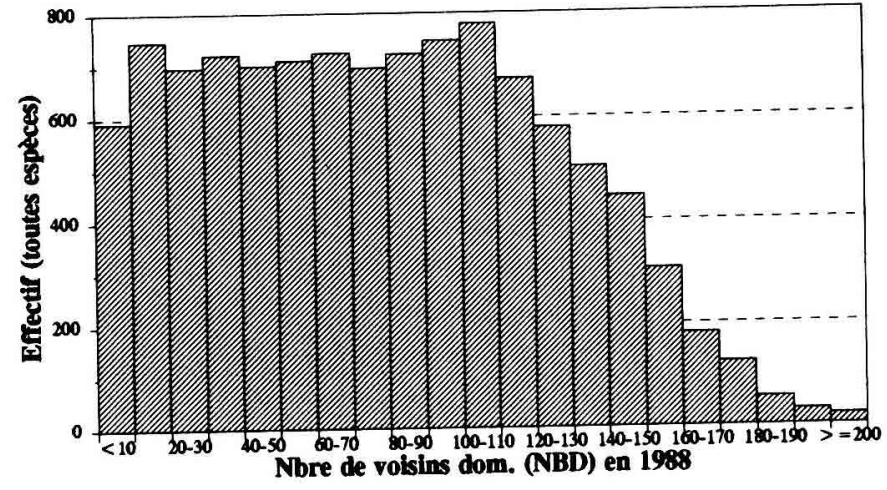

(d)

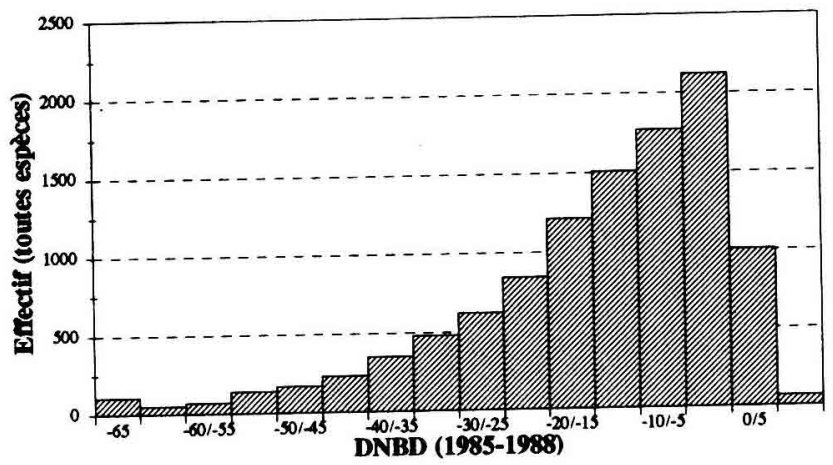

(e)

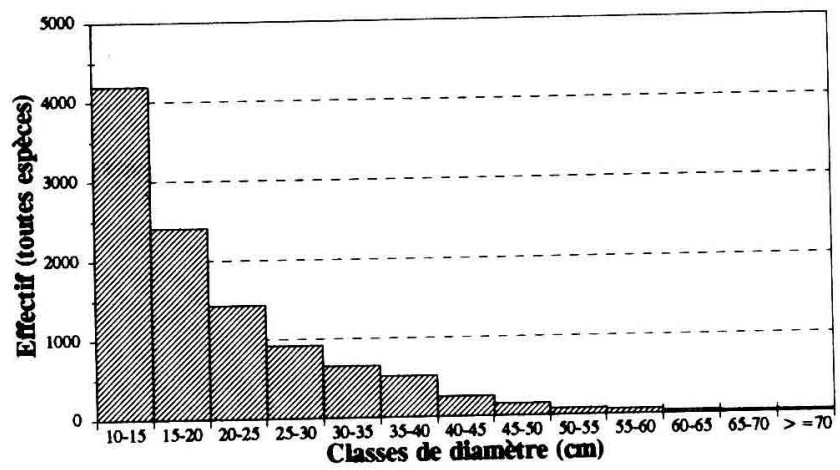


(a)

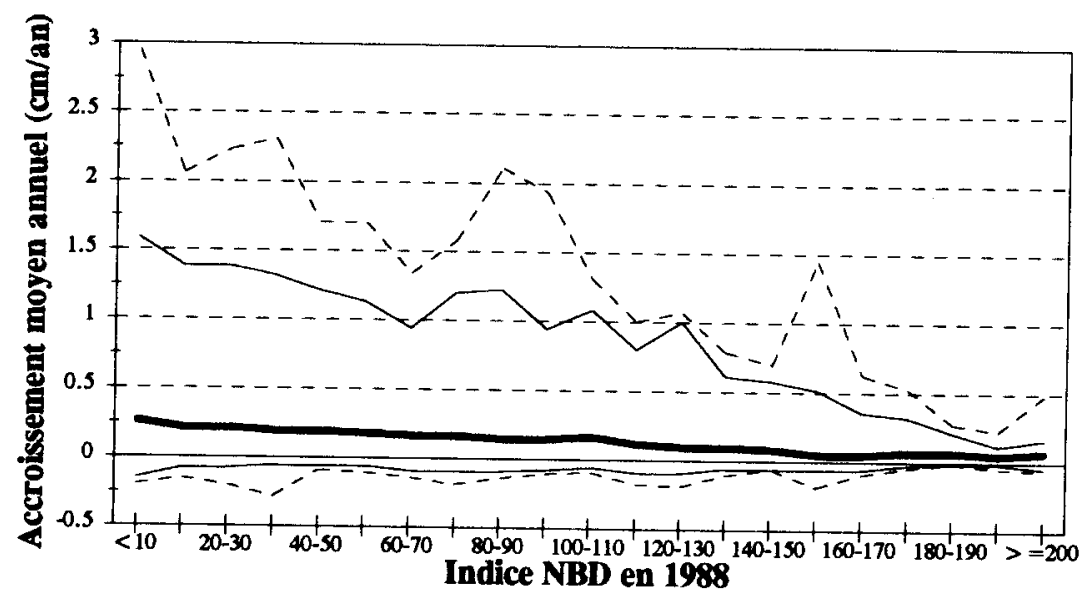

- - Mini/Maxi — Mini-4/Maxi-4 Médiane

(b)

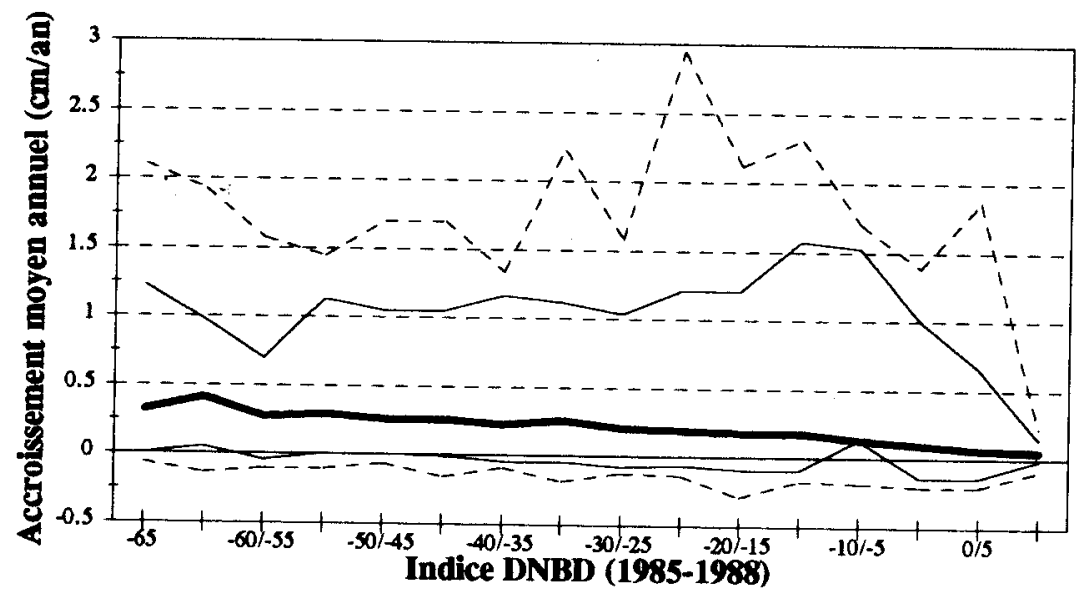

- - Mini/Maxi - Mini-4/Maxi-4 - Médiane 


\section{(c)}

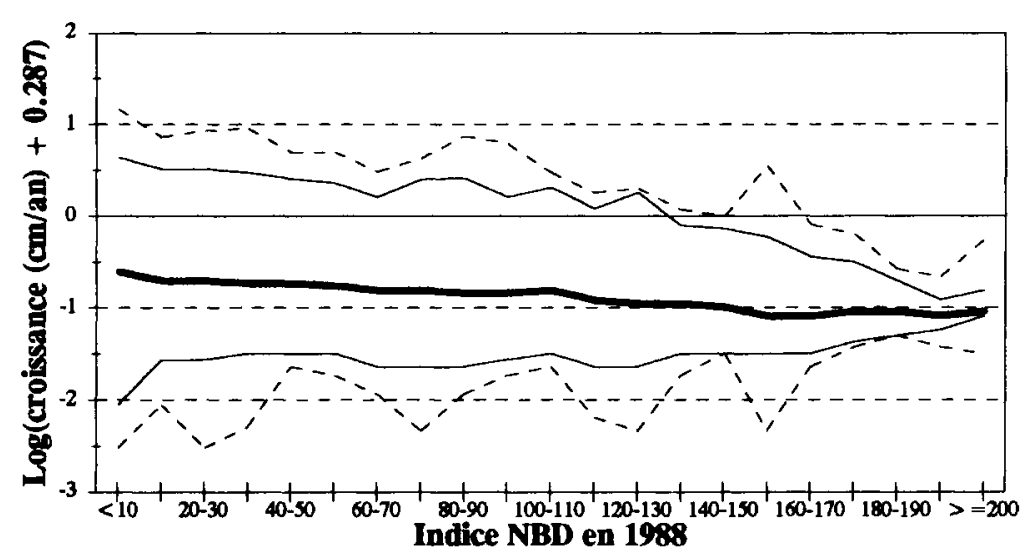

- - Mini/Maxi - Mini-4/Maxi-4 Médiane

(d)

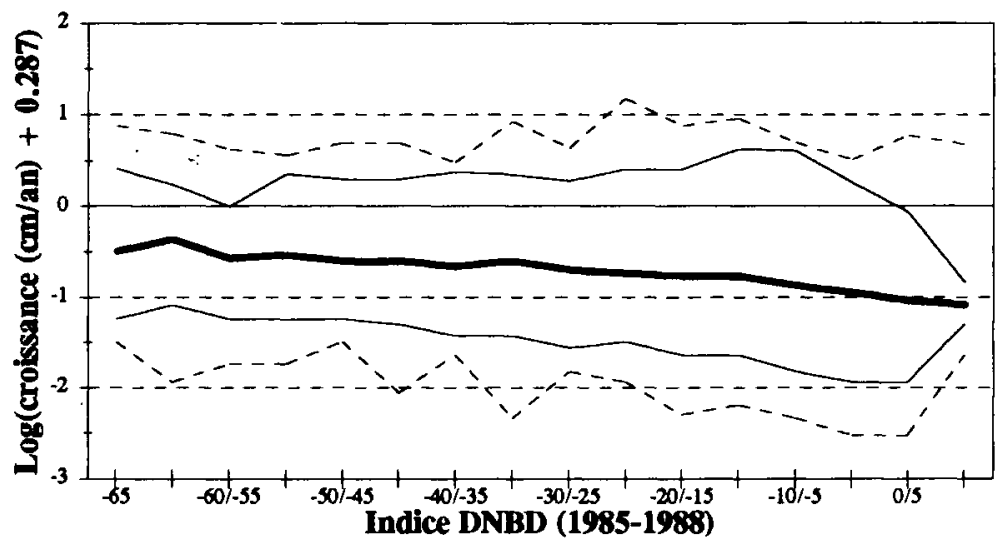

\section{-- Mini/Maxi $\quad$ Mini-4/Maxi-4 - Médiane}

Figure 2. Accroissement moyen annuel (lissé sur trois ans, entre 1988 et 1991) sur les huit parcelles d'étude, représenté en fonction de deux indices de compétition calculés sur des placettes de $30 \mathrm{~m}$ de rayon : nombre de voisins dominants (a), et évolution du nombre des voisins dominants entre 1988 et 1991 (b). Même chose pour respectivement (c) et (d) en prenant Log (accroissement $+0,287$ ) en ordonnée. Mini-4 (resp. Maxi-4) désigne la valeur de l'accroissement la plus faible (resp. la plus élevée) une fois que les quatre points extrêmes ont été éliminés. 
Cette dernière opération a également été réalisée avec des tranches de superficie constante (donc de largeur décroissante : voir figure $3 b$ ), pour pallier le biais éventuel dû au fait que l'ajout à la placette initiale de tranches successives de largeur égale donc de superficie croissante, se traduisait par l'ajout d'un nombre, lui aussi croissant, d'arbres au stock de départ.

\section{RÉSULTATS ET DISCUSSION}

\subsection{Influence du diamètre sur les accroissements}

Toutes espèces confondues, sur les seules parcelles témoins, le coefficient de corrélation linéaire de Pearson entre le diamètre $D$ des arbres et le logarithme de leur accroissement moyen annuel en dia- mètre sur trois ans $\log (\Delta D+c)$ est faible : $r=0,21(p<0,0001)$. Ce résultat rejoint les observations de Alder [1] et peut s'interpréter ainsi : les petits arbres ont certes une probabilité importante d'appartenir à une espèce de «sousbois », d'être dominés, d'avoir un houppier très peu éclairé et donc une croissance lente, mais ce sont aussi ceux qui appartiennent souvent à "l'ensemble d'avenir » (sensu [29] : individus n'ayant pas encore subi de réitération et n'ayant pas atteint la taille maximale), et qui peuvent voir leur croissance augmenter de manière considérable dès que les conditions deviennent favorables. À l'inverse, les gros arbres qui font partie des ensembles structuraux supérieurs et ont toute chance de se trouver dans des conditions favorables (co-dominants ou dominants, houppiers bien éclairés) sont

Tableau III. Effet des traitements sylvicoles sur la valeur des coefficients de corrélation entre quelques indices de compétition calculés sur des placettes de rayon $30 \mathrm{~m}$, et le logarithme de l'accroissement moyen annuel en diamètre des arbres sujets (10 745 individus) : on a fait figurer respectivement le coefficient de corrélation linéaire de Pearson et le coefficient de corrélation de rang de Spearman entre parenthèses. Toutes les espèces sont confondues.

\begin{tabular}{|c|c|c|c|c|c|c|}
\hline Traitement & NBT & STT & NBD & STD & & \\
\hline Témoin & $-0,12(-0,12)$ & $-0,14(-0,11)$ & $-0,28(-0,29)$ & $-0,26(-0,25)$ & & \\
\hline $\mathrm{T} 1$ & $-0,23(-0,22)$ & $-0,20(-0,18)$ & $-0,22(-0,22)$ & $-0,21(-0,23)$ & & \\
\hline $\mathrm{T} 2$ & $-0,20(-0,21)$ & $-0,12(-0,13)$ & $-0,21(-0,21)$ & $-0,18(-0,19)$ & & \\
\hline T3 & $-0,23(-0,23)$ & $-0,22(-0,22)$ & $-0,25(-0,24)$ & $-0,26(-0,25)$ & & \\
\hline \multirow[t]{2}{*}{ Tous } & $-0,26(-0,26)$ & $-0,29(-0,30)$ & $-0,25(-0,26)$ & $-0,31(-0,33)$ & & \\
\hline & DNBT & DSTT & DNBD & DSTD & $\mathbf{R}^{2 *}$ & $\sigma^{* *}$ \\
\hline Témoin & NS (NS) & NS (NS) & $-0,33(-0,26)$ & $-0,12(-0,19)$ & 0,24 & $\mathbf{0 , 3 0}$ \\
\hline $\mathrm{T} 1$ & $-0,11(-0,11)$ & $-0,17(-0,17)$ & $-0,18(-0,14)$ & $-0,21(-0,20)$ & 0,18 & $\mathbf{0 , 3 4}$ \\
\hline $\mathrm{T} 2$ & $-0,12(-0,14)$ & $-0,15(-0,15)$ & $-0,23(-0,2 I)$ & $-0,19(-0,18)$ & 0,17 & 0,41 \\
\hline T3 & $-0,10(-0,09)$ & $-0,15(-0,15)$ & $-0,09(-0,08)$ & $-0,15(-0,15)$ & 0,13 & $\mathbf{0 , 3 7}$ \\
\hline Tous & $-0,25(-0,26)$ & $-0,28(-0,30)$ & $-0,29(-0,32)$ & $-0,30(-0,32)$ & 0,20 & $\mathbf{0 , 3 6}$ \\
\hline
\end{tabular}

NB. NS: coefficient non significativement différent de $0(p \geq 0,05)$. Les autres coefficients sont tous significativement non nuls $(p \leq 0,0001)$. Coefficient de détermination, traduisant la part de variance du logarithme de l'accroissement expliquée par la prise en compte, dans un modèle linéaire, des deux variables «explicatives» indiquées en italique gras dans chaque traitement. ** Estimation de l'écart-type résiduel correspondant à ce modèle $(\log (\mathrm{cm}))$. 
aussi ceux qui appartiennent aux ensembles architecturaux du présent ou du passé (toujours sensu Hallé et Oldeman, [29] : individus ayant atteint leur taille maximale à la suite de nombreuses réitérations, sénescents, ou dégénérés à la suite dun traumatisme) et dont la croissance ne va pas tarder à stagner.

Lorsque toutes les parcelles, témoins et traitées, sont prises en compte dans le calcul, le coefficient de corrélation linéaire chute à $r=0,12(p<0,0001)$. Dans un milieu qui change très peu et très lentement, comme c'est le cas en forêt naturelle intouchée, le diamètre d'un arbre peut être considéré comme un indicateur (certes peu efficace) de conditions de croissance passées ; ces conditions évoluant peu, il est normal que le diamètre soit également corrélé aux accroissements ultérieurs si la période considérée nest pas trop longue. En revanche, dès qu'interviennent une exploitation et/ou un traitement sylvicole qui modifient soudainement et de façon importante l'environnement des arbres, le diamètre perd son potentiel de prédiction et la recherche d'autres indices prend toute son importance.

La variable D n'a par la suite plus été considérée, car elle est liée linéairement à l'indice NBD ( $r=0,84$, lorsque les placettes de calcul font $30 \mathrm{~m}$ de rayon).

\subsection{Efficacité des différents indices étudiés, en fonction des traitements sylvicoles ou tous traitements confondus}

Les résultats obtenus toutes espèces et tous diamètres confondus pour des placettes de rayon $30 \mathrm{~m}$ figurent dans le tableau III. Ils montrent que les indices indépendants du diamètre de l'arbre sujet (NBT, DNBT, STT, DSTT) sont le plus souvent un peu moins performants que les autres, tout particulièrement dans les parcelles témoins où la densité est forte et la compétition pour la lumière importante. Les variations d'indice sont souvent des prédicteurs aussi efficaces que les indices eux-mêmes. À noter que tous ces indices sont plus ou moins liés entre eux, mais qu'en général la liaison entre indice (a)

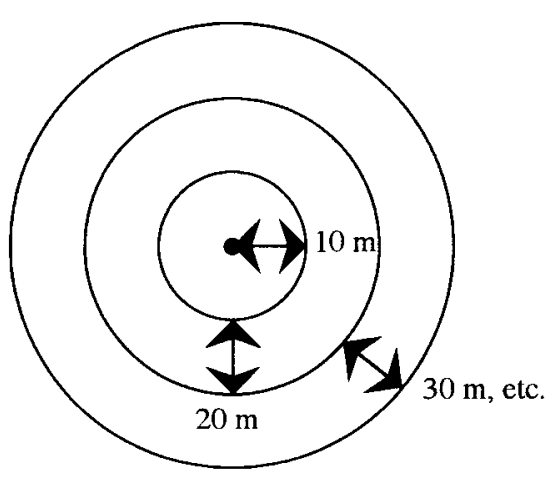

(b)

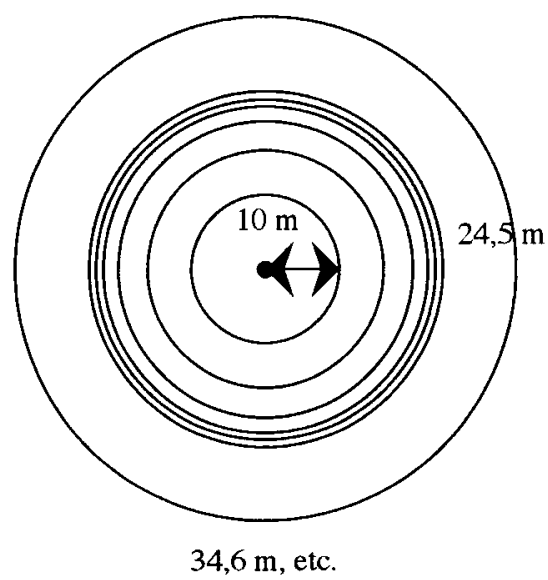

Figure 3. Tranches successives utilisées pour la comparaison de modèles emboîtés. (a) Amplitude constante. (b) Superficie constante. 
statique et indice dynamique correspondant est faible : $r \approx 0,2$ entre IC et DIC.

Étant données les périodes de calcul choisies pour les différentes variables, l'évolution des indices intègre les traitements sylvicoles et la croissance traduit la réaction du peuplement à ces traitements. Si aucune tendance nette ne se dégage du côté des indices statiques on observe que, à quelques exceptions près, plus le traitement est fort moins les indices dynamiques paraissent efficaces et cette dégradation des résultats transparaît clairement dans la valeur du coefficient de détermination. Cela signifie que la variation brutale de l'environnement des arbres dans les parcelles traitées est moins corrélée à leur réaction en croissance qu'une variation plus progressive en parcelles témoins. Cette observation pourrait s'expliquer par la réaction «négative " de certains sujets à une mise en lumière trop brusque et à une modification trop importante du microclimat local à la suite des traitements, tout particulièrement dans le $\mathbf{n}^{\circ} 3$.

Quel que soit le traitement sylvicole, et tous traitements confondus, le meilleur modèle linéaire permettant de prédire la croissance s'est révélé être :

$$
\log (\Delta D+c)=a^{*} N B D+b^{*} \text { DNBD }+d
$$

Ce résultat reste vrai pour toutes les tailles de placettes étudiées (voir tableau $V$. Pour des placettes de $30 \mathrm{~m}$ de rayon (10745 arbres dans le jeu de données), a $=0,0031, \mathrm{~b}=0,0104, \mathrm{~d}=0,6992$ (coefficients significativement non nuls), et le modèle prend en compte $21 \%$ de la variabilité observée $(\sigma=0,356)$. La constante c vaut 0,287 , valeur choisie supérieure au plus petit accroissement négatif observé dans le jeu de données.

\subsection{Influence du diamètre sur les liaisons indices de compétition / accroissement}

Nous avons travaillé toutes espèces et tous traitements sylvicoles confondus, sur des placettes de rayon $10 \mathrm{~m}$ et $30 \mathrm{~m}$, mais les tendances étant comparables nous n'avons fait figurer les résultats que pour les placettes de grande taille. Les arbres ont été ventilés en classes de diamètre de $5 \mathrm{~cm}$ d'amplitude, la dernière classe regroupant tous les individus de plus de $60 \mathrm{~cm}$. Les coefficients de corrélation linéaire obtenus entre les différents indices de compétition et l'accroissement ont été reportés dans le tableau $I V$, et représentés sur les graphiques de la figure 4. Ils font apparaître que :

- i) la valeur des coefficients de corrélation décroît lorsque le diamètre des arbres sujets augmente, jusqu'à la classe $30-35 \mathrm{~cm}$ pour laquelle on observe les valeurs les plus faibles. Puis elle ré-augmente, avant de devenir non significative pour pratiquement tous les indices à partir de la classe $60 \mathrm{~cm}$ et plus. À noter qu'à partir de $50 \mathrm{~cm}$ de diamètre, les effectifs deviennent faibles dans les classes et les résultats erratiques et peu fiables ;

- ii) les indices dépendant du diamètre (NBD et STD) ne sont plus significativement corrélés à l'accroissement à partir des classes de diamètre $50-55 \mathrm{~cm}$ (dès $40-45 \mathrm{~cm}$ pour les placettes de rayon $10 \mathrm{~m}$ ), contrairement aux simples indices de densité locale (NBT, STT). STT donne le plus souvent de meilleurs résultats que les autres indices statiques. En revanche, dans pratiquement tous les cas, les variations d'indice DNBD et DSTD sont davantage liées à l'accroissement que DNBT et DSTT, DNBD étant de loin le plus « efficace ». 
La classe $30-40 \mathrm{~cm}$ constitue une classe charnière dans ce type de forêt. En effet, c'est dans cette gamme de taille que les arbres appartenant aux espèces des strates supérieures passent dans la voûte forestière, et que les arbres appartenant à de nombreuses espèces des strates inférieures atteignent leur taille maximale. La diversité des situations rencontrées est très grande: arbres dominés, codominants, dominants, de tempéraments divers, ce qui peut expliquer la mauvaise performance générale observée sur les indices.

Dans les classes plus petites, les arbres subissent une concurrence importante pour la lumière et sans doute également pour l'alimentation en eau et en substances nutritives : la performance des différents indices NBT, STT, NBD et
STD est très comparable. La disparition, ou l'apparition d'individus dominants influe beaucoup sur la croissance ce qui explique l'efficacité de la variable DNBD. À noter que dans ces gammes de taille, les variables STT, STD d'une part et DSTT, DSTD d'autre part ont des valeurs très proches : leurs performances sont tout à fait comparables.

Dans les classes supérieures (à partir surtout de la classe $50-55 \mathrm{~cm}$ ), la compétition pour la lumière devient en théorie beaucoup moins importante, alors qu'elle l'est toujours pour les autres facteurs de croissance. Les simples indices de densité locale donnent de meilleurs résultats que les indices $\mathrm{NBD}$ et $\mathrm{STD}$, liés au statut social. Cependant, on observe toujours des arbres dominés ou co-dominants donc gênés dans leur accès

Tableau IV. Influence de la classe de diamètre sur les coefficients de corrélation linéaire (Pearson) calculés entre les indices de compétition étudiés et le logarithme de l'accroissement moyen annuel en diamètre des arbres sujets. Rayon des placettes : $30 \mathrm{~m}$ (10 745 individus). Toutes les espèces sont confondues.

Classes de Effectif NBT DNBT STT DSTT NBD DNBD STD DSTD R2* $\sigma^{* *}$ diamètre

\begin{tabular}{|c|c|c|c|c|c|c|c|c|c|c|c|}
\hline $10-15$ & 4197 & $-0,32$ & $-0,31$ & $-0,34$ & $-0,35$ & $-0,32$ & $-0,46$ & $-0,34$ & $-0,37$ & 0,28 & $\mathbf{0 , 3 1}$ \\
\hline $15-20$ & 2407 & $-0,27$ & $-0,24$ & $-0,31$ & $-0,31$ & $-0,22$ & $-0,53$ & $-0,30$ & $-0,36$ & 0,36 & 0,32 \\
\hline $20-25$ & 1436 & $-0,14$ & $-0,16$ & $-(0,21$ & $-0,19$ & $-0,20$ & $-0,38$ & $-0,21$ & $-0,23$ & 0,20 & $\mathbf{0 , 4 0}$ \\
\hline $25-30$ & 920 & $-0,21$ & $-0,24$ & $-0,27$ & $-0,26$ & $-0,22$ & $-0,43$ & $-0,25$ & $-0,30$ & 0,24 & $\mathbf{0 , 3 5}$ \\
\hline $30-35$ & 657 & $-0,16$ & $-0,12$ & $-0,21$ & $-0,19$ & $-0,22$ & $-0,33$ & $-0,20$ & $-0,24$ & 0,16 & $\mathbf{0 , 3 8}$ \\
\hline $35-40$ & 517 & $-0,20$ & $-0,19$ & $-0,27$ & $-0,22$ & $-0,23$ & $-0,38$ & $-0,23$ & $-0,28$ & 0,21 & $\mathbf{0 , 3 8}$ \\
\hline $40-45$ & 250 & $-0,21$ & $-0,30$ & $-0,29$ & $-0,33$ & $-0,27$ & $-0,4 I$ & $-0,25$ & $-0,35$ & 0,18 & $\mathbf{0 , 3 8}$ \\
\hline $45-50$ & 150 & $-0,24$ & $-0,35$ & $-0,35$ & $-0,44$ & $-0,33$ & $-0,47$ & $-0,31$ & $-0,46$ & 0,23 & 0,42 \\
\hline $50-55$ & 73 & $-0,34$ & $-0,23$ & $-0,33$ & $-0,32$ & NS & $-0,43$ & NS & $-0,44$ & 0,23 & 0,49 \\
\hline $55-60$ & 61 & $-0,33$ & $-0,29$ & $-0,37$ & $-0,31$ & NS & $-0,34$ & $-0,27$ & $-0,31$ & 0,16 & 0,43 \\
\hline$\geq 60$ & 77 & $-0,26$ & NS & $-0,26$ & NS & NS & NS & NS & NS & 0,07 & 0,43 \\
\hline $\begin{array}{l}\text { Tous } \\
\text { diamètres }\end{array}$ & 0745 & $-0,26$ & $-0,25$ & $-0,29$ & $-0,28$ & $-0,25$ & $-0,29$ & $-0,31$ & $-0,30$ & 0,20 & 0,36 \\
\hline
\end{tabular}

NB. NS : coefficient non significativement différent de $0(p \geq 0,(05)$. Les autres coeflicients sont tous significativement non nuls $(p \leq 0,0001)$.

* Coefficient de détermination, traduisant la part de variance du logarithme de la croissance expliquée par la prie en compte, dans un modèle linéaire, des deux variables «explicatives» indiquées en italique gras dans chaque traitement. ** Estimation de l'écart-type résiduel correspondant à ce modèle $(\log (\mathrm{cm}))$. 
(a)

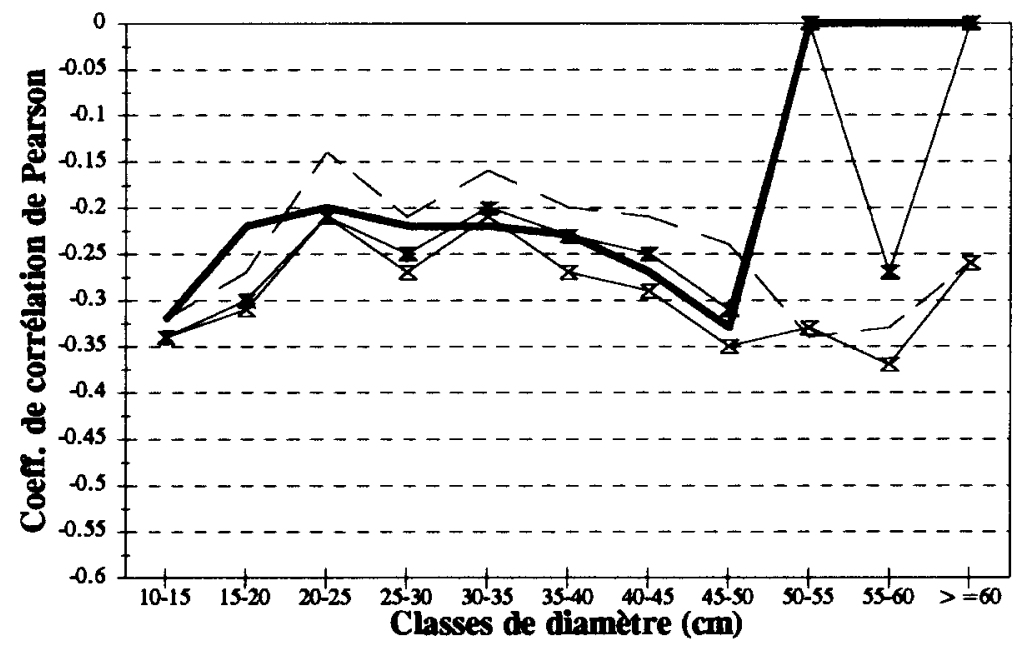

(b)

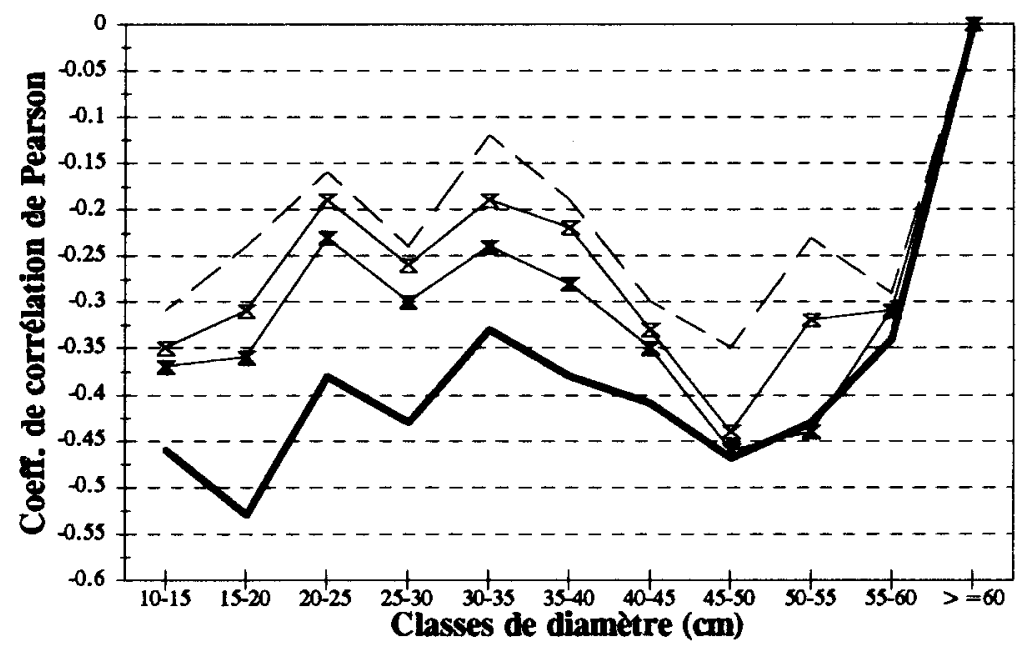

\section{-- DNBT $-z$ DSTT - DNBD - DSTD}

Figure 4. Influence de la classe de diamètre sur les coefficients de corrélation linéaire entre différents indices de compétition calculés sur des placettes de rayon $30 \mathrm{~m}$ et le logarithme de l'accroissement moyen annuel en diamètre des arbres sujets. Toutes les espèces sont confondues. 
à la lumière, dans ces gammes de diamètre (en moyenne, 0,5 voisins de taille supérieure pour des arbres de la classe 50-55 cm dans un rayon de $10 \mathrm{~m}$, un peu plus de quatre dans un rayon de $30 \mathrm{~m}$ ) : la bonne performance des variables DNBD et DSTD peut s'expliquer par l'existence d'une forte capacité de réaction, grâce aux phénomènes de réitération (arbres du présent, voir plus haut), à toute augmentation de la ressource lumineuse au niveau des houppiers. Cependant, au-delà de $55-60 \mathrm{~cm}$ de diamètre, les arbres ont, pour la plupart, perdu ces facultés d'adaptation et les variations d'indices ne sont plus liés à leur accroissement.

Le meilleur modèle linéaire à deux variables permettant de décrire la croissance fait pratiquement toujours intervenir DNBD, en association avec d'autres indices dynamiques : DNBT dans les petites classes de diamètre et DSTT dans les classes supérieures. C'est pour les petites classes de diamètre (de 10 à $30 \mathrm{~cm}$ ) que ce type de modèle est le plus efficace.

\section{Remarque sur les interactions}

Les interactions évidentes existant entre le diamètre et les indices de compétition peuvent être prises en compte à travers l'introduction, dans le modèle $\log (\Delta \mathrm{D}+\mathrm{c})=\mathrm{f}(\mathrm{IC})$ d'un terme d'interaction $D^{*} I C$. Si l'on recherche de manière systématique les deux variables les plus efficaces parmi 90 variables constituées de nos huit indices de base et du diamètre, des monômes du deuxième degré correspondants et de tous les termes d'interaction pris deux à deux, ce sont lindice NBD et le terme D*DNBD qui se retrouvent sélectionnés. Ce dernier terme traduit la nécessité, pour obtenir un effet positif sur les accroissements, d'éliminer d'autant plus d'arbres dans le voisinage d'un sujet que celui-ci est petit. Il traduit, à l'inverse, l'effet très négatif de l'appari- tion d'un individu dominant dans le voisinage d'un gros arbre (plus gênant que dans le voisinage d'un petit arbre). Le modèle utilisant ces deux variables est alors caractérisé par un coefficient de détermination de $0,24(\sigma=0,348)$.

Cependant, l'utilisation du terme D*DNBD dans un modèle plus complexe, traduisant à la fois les potentialités propres de l'individu, et l'effet régulateur de l'environnement perd de son intérêt au regard de la variable DNBD. Nous l'avons donc provisoirement écarté.

\subsection{Recherche d'un rayon optimal pour les placettes de calcul}

\subsection{1. Évolution des liaisons indices de compétition/accroissement avec le rayon des placettes de calcul}

Nous avons retenu 8609 individus situés à plus de $40 \mathrm{~m}$ des bords des huit parcelles d'étude et fait varier le rayon des placettes entre 10 et $40 \mathrm{~m}$ par pas de $5 \mathrm{~m}$. Les résultats toutes classes de diamètre et toutes espèces confondues figurent dans le tableau $V$, et sont représentés figure $5 a$ et $5 b$. Sur cette même figure ont également été représentés pour comparaison les résultats obtenus sur les deux premières classes de diamètre.

Pratiquement tous les coefficients de corrélation augmentent avec la taille des placettes, jusqu'à $20 \mathrm{~m}$ de rayon, puis se stabilisent. On observe la même tendance, à une exception près (variable NBD pour la classe de diamètre 15-20 $\mathrm{cm}$ ) en ne considérant que les petites classes de diamètre (voir figure $5 c, 5 d, 5 e$ et $5 f$ ), pour lesquelles on note toujours la nette supériorité de la variable DNBD. Tous diamètres confondus, le meilleur modèle linéaire à deux variables fait intervenir NBD et DNBD dans tous les cas. Le coefficient de détermination se stabilise à 0,20 à partir de placettes de rayon $25 \mathrm{~m}$. 


\subsection{2. Étude de l'apport de «tranches successives» à des placettes initiales de $10 \mathrm{~m}$ de rayon}

L'un des problèmes posés par les résultats du tableau $V$ est le suivant : les différences relativement faibles observées entre coefficients de corrélation sont-elles significatives, autrement dit est-il réellement indispensable de calculer les indices sur des placettes de rayon supérieur à $10 \mathrm{~m}$ ?

Pour répondre à cette question, seuls les deux indices les plus efficaces toutes classes de diamètre et toutes espèces confondues quelle que soit la taille de la placette, ont été pris en considération : soit NBD et $\triangle D N B D$. Nous avons étendu le rayon des placettes jusqu'à $50 \mathrm{~m}$, ne conservant qu'un jeu de 6631 arbres tous situés à plus de $50 \mathrm{~m}$ des bords des huit parcelles, et nous avons procédé à l'étude des modèles emboîtés : les résultats concernant des tranches suc- cessives de largeur constante figurent dans le tableau VI. Si l'on fixe le risque d'erreur à $5 \%$, on constate que chaque ajout d'information améliore le résultat global de manière très significative (particulièrement net en ce qui concerne le passage de $0-10 \mathrm{~m}$ à $0-20 \mathrm{~m}$ ), et ce jusqu'à $50 \mathrm{~m}$ mais avec une valeur du $\mathrm{F}$ en diminution rapide. Si l'on inclut tous les indices dans le même modèle $\left(\mathrm{R}^{2}=\right.$ $0,24)$, on observe que jusqu'à $20 \mathrm{~m}$ les coefficients de NBD et de DNBD sont significativement non nuls, alors qu'audelà, seule l'information apportée par l'indice dynamique DNBD reste utile. Les résultats obtenus en considérant des tranches successives de superficie constante sont tout à fait comparables aux précédents.

Comment expliquer ces différentes observations ? Il faut se souvenir que l'indice NBD est avant tout un indice de statut social. Un arbre dominé par son

Tableau V. Influence du rayon des placettes sur les coefficients de corrélation linéaire (Pearson) calculés entre les indices de compétition étudiés et le logarithme de l'accroissement moyen annuel en diamètre des arbres sujets. Toutes classes de diamètre et toutes espèces confondues (8 609 individus étudiés).

Rayon des

placettes (m) NBT DNBT STT DSTT NBD DNBD STD DSTD R ${ }^{2 *} \sigma^{* *}$

$\begin{array}{lllllllllll}10 & -0,21 & -0,19 & -0,22 & -0,19 & \mathbf{- 0 , 2 6} & \mathbf{- 0 , 2 5} & -0,25 & -0,20 & \mathbf{0 , 1 4} & \mathbf{0 , 3 8} \\ 15 & -0,24 & -0,22 & -0,27 & -0,24 & \mathbf{- 0 , 2 7} & \mathbf{- 0 , 2 8} & -0,30 & -0,26 & \mathbf{0 , 1 7} & \mathbf{0 , 3 7} \\ 20 & -0,24 & -0,23 & -0,28 & -0,26 & \mathbf{- 0 , 2 6} & \mathbf{- 0 , 2 8} & -0,31 & -0,28 & \mathbf{0 , 1 9} & \mathbf{0 , 3 7} \\ 25 & -0,25 & -0,23 & -0,28 & -0,28 & \mathbf{- 0 , 2 6} & \mathbf{- 0 , 2 9} & -0,31 & -0,30 & \mathbf{0 , 2 0} & \mathbf{0 , 3 7} \\ 30 & -0,25 & -0,23 & -0,28 & -0,28 & \mathbf{- 0 , 2 5} & \mathbf{- 0 , 2 8} & -0,31 & -0,30 & \mathbf{0 , 2 0} & \mathbf{0 , 3 7} \\ 35 & -0,25 & -0,24 & -0,28 & -0,28 & \mathbf{- 0 , 2 5} & \mathbf{- 0 , 2 8} & -0,31 & -0,30 & \mathbf{0 , 2 0} & \mathbf{0 , 3 6} \\ 40 & -0,25 & -0,24 & -0,29 & -0,28 & \mathbf{- 0 , 2 5} & \mathbf{- 0 , 2 8} & -0,31 & -0,30 & \mathbf{0 , 2 0} & \mathbf{0 , 3 6}\end{array}$

NB. Les coefficients sont tous significativement non nuls $(p \leq 0,0001)$. ${ }^{*}$ Coefficient de détermination, traduisant la part de variance du logarithme de la croissance expliquée par la prise en compte, dans un modèle linéaire, des deux variables «explicatives» indiquées en italique gras dans chaque traitement. ** Estimation de l'écart-type résiduel correspondant à ce modèle $(\log (\mathrm{cm})$. 
entourage proche l'est tout autant par son entourage lointain, de même un arbre dominant son entourage proche «domine» son entourage lointain : il n'est ainsi pas étonnant que les liaisons accroissement/NBD varient peu avec l'éloignement, et qu'au-delà d'une certaine distance (en l'occurrence $20 \mathrm{~m}$ ) l'information supplémentaire apportée par cette variable n'ait plus d'intérêt. En ce qui concerne DNBD, cet indice traduit une augmentation ou une diminution de la ressource (tous types de ressource et particulièrement ressource lumineuse) dans l'entourage des arbres. Il semblerait que ceux-ci soient encore sensibles à des variations se produisant à grande distance, sans qu'il soit vraiment possible de trancher sur cette distance. Un seul fait paraît assez clair : ce rayon doit être supérieur ou égal à $20 \mathrm{~m}$.
Stone et Kalisz [62] ont recensé un grand nombre de travaux réalisés sur l'extension radiale des racines d'arbres isolés ou en peuplement, tant en forêt tempérée que tropicale: ils signalent que les valeurs les plus fréquemment rapportées pour cette variable se situent entre 10 et $20 \mathrm{~m}$, mais que l'effet sur des cultures intercalaires ou avoisinantes atteint $30 \mathrm{~m}$. En Guyane française, Atger et Edelin [4] travaillant sur l'architecture des systèmes racinaires de différentes espèces notent une extension latérale dépassant $10 \mathrm{~m}$ chez Cecropia obtusa (espèce pionnière atteignant environ $30 \mathrm{~cm}$ de diamètre à $1,30 \mathrm{~m}$ ) et Roggy (communication personnelle), a suivi les racines d'un Sterculia pruriens de $110 \mathrm{~cm}$ de diamètre sur plus de $50 \mathrm{~m}$. À noter que nous avons toujours admis de manière implicite que l'extension racinaire des arbres, étroitement liée à la notion d'« espace

Tableau VI. Comparaison de modèles emboîtés. À chaque étape, on ajoute au modèle linéaire global les deux indices NBD et DNBD correspondant à la «tranche» de distance suivante, et on teste l'utilité de cet apport d'information. Toutes classes de diamètre et toutes espèces confondues (6 631 individus étudiés).

\begin{tabular}{|c|c|c|c|c|}
\hline $\begin{array}{l}\text { «Tranche » de } \\
\text { distance }(\mathrm{m})\end{array}$ & $\begin{array}{l}\text { Indices pris } \\
\text { en compte }\end{array}$ & $\mathrm{R}^{2}$ du modèle & $\mathrm{F}^{*}$ & $\begin{array}{l}\text { Coefficient des } \\
\text { indices dans } \\
\text { le modèle complet ** }\end{array}$ \\
\hline $0-10$ & $\begin{array}{c}\text { NBD } \\
\text { DNBD }\end{array}$ & 0,15 & & $\begin{array}{l}S \\
S\end{array}$ \\
\hline $10-20$ & $\begin{array}{c}\text { NBD } \\
\text { DNBD }\end{array}$ & 0,20 & 224,6 & $\begin{array}{l}S \\
S\end{array}$ \\
\hline $20-30$ & $\begin{array}{l}\text { NBD } \\
\text { DNBD }\end{array}$ & 0,22 & 68,5 & $\begin{array}{c}\text { NS } \\
\text { S }\end{array}$ \\
\hline $30-40$ & $\begin{array}{l}\text { NBD } \\
\text { DNBD }\end{array}$ & 0,23 & 45,5 & NS \\
\hline $40-50$ & $\begin{array}{c}\text { NBD } \\
\text { DNBD }\end{array}$ & 0,24 & 29,2 & $\begin{array}{c}\text { NS } \\
\text { S }\end{array}$ \\
\hline
\end{tabular}

\footnotetext{
* $\mathrm{F}_{\lim (2, \infty) \mathrm{ddl}}=3,00$ pour un risque d'erreur de $5 \%$.

** S : valeur significativement différente de $0(p \leq 0,0001)$, toujours négative. NS : valeur non significativement différente de $0(p \geq 0,05)$.
} 
(a)

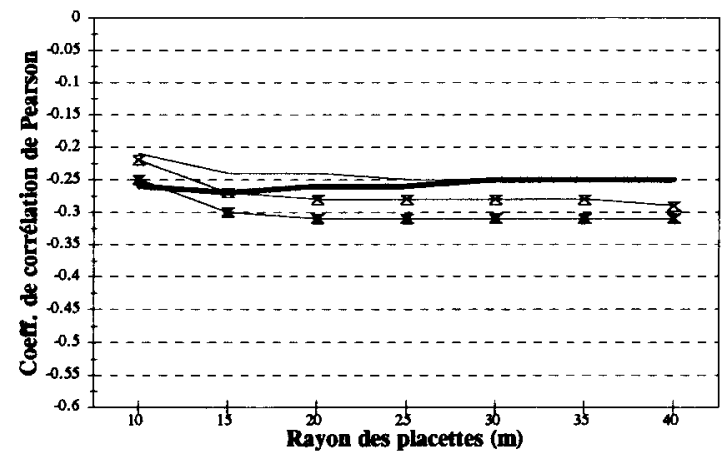

- NBT $¥$ STT - NBD $\rightarrow$ STD

(b)

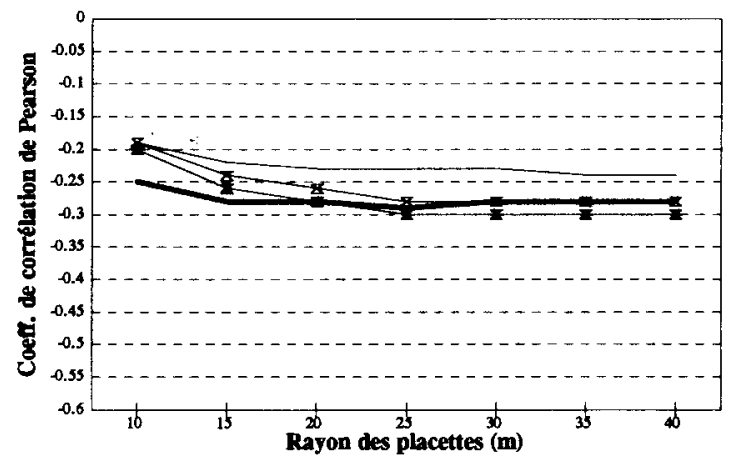

DNBT \&-DSTT —DNBD —- DSTD

(c)

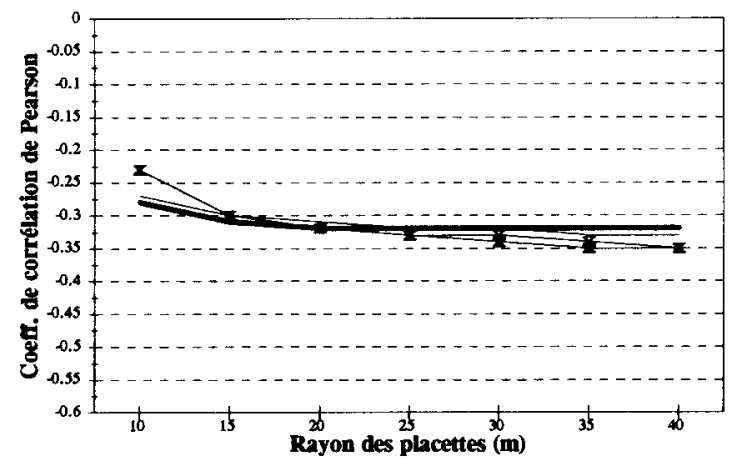

NBT ₹-STT - NBD - STD 
(d)

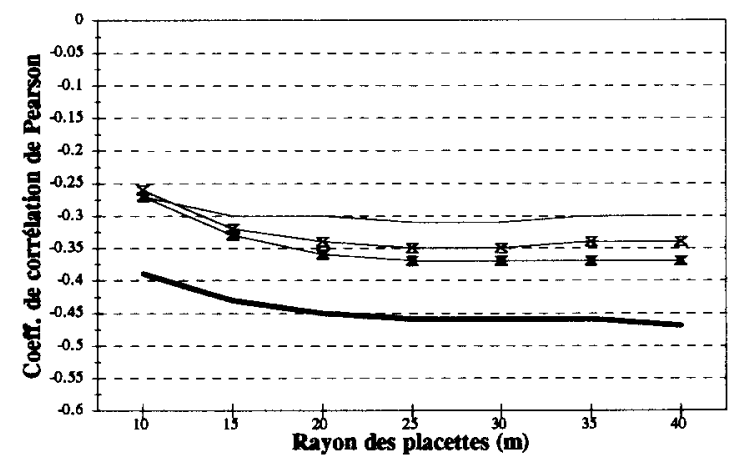

- DNBT — DSTT - DNBD $=$ DSTD

(e)

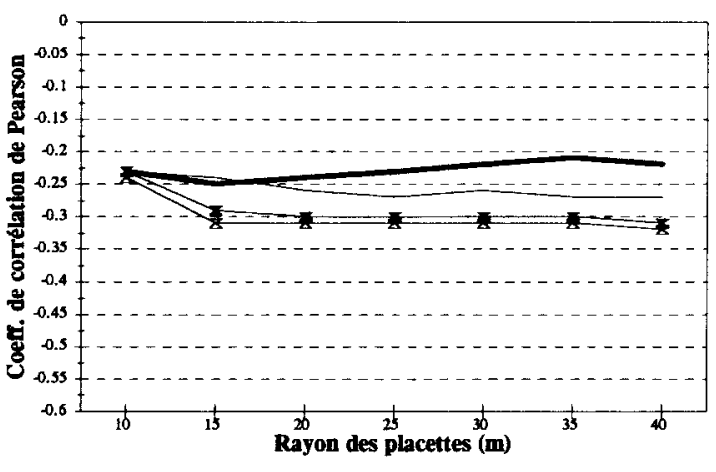

- NBT $\&$ STT $\longrightarrow$ NBD $=$ - STD

(f)

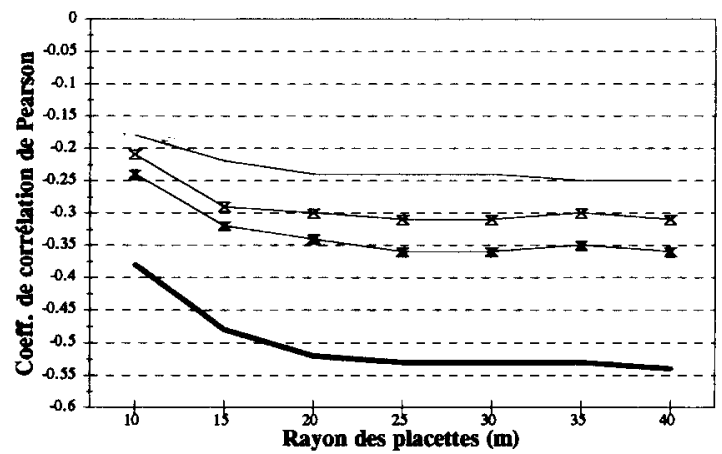

DNBT $\approx$ DSTT - DNBD - DSTD
Figure 5. Influence du rayon des placettes de calcul sur les coefficients de corrélation linéaire (Pearson) entre différents indices de compétition et le logarithme de l'accroissement moyen annuel en diamètre des arbres sujets, toutes espèces confondues. (a) et (b) Tous diamètres confondus. (c) et (d) Arbres de la classe de diamètre $10-15 \mathrm{~cm}$. (e) et (f) Arbres de la classe de diamètre $15-20 \mathrm{~cm}$. 
vital " et à la superficie des placettes d'étude, était isotrope, ce qui est vraisemblablement faux et explique sans doute en partie que les résultats observés soient aussi peu concluants.

\subsection{Influence de l'information « espèce » sur les liaisons accroisse ment / indices de compétition}

Les études de ce type se heurtent rapidement au problème des faibles effectifs, dès que l'on considère séparément chaque espèce : le choix se trouve assez vite limité, lorsqu'en particulier on veut retenir des comportements contrastés. Il n'a pas été possible, par exemple, d'étudier d'espèces pionnières trop peu abondantes dans les parcelles.

Six espèces ont été retenues : Bocoa prouacensis Aublet (Boco), Carapa procera A., de Candolle (Carapa), Dicorynia guianensis (Aubl.) Amshoff (Angélique), première espèce exploitée en Guyane, Licania micrantha Miq., Sloanea guianensis (Aubl.) Benth et Symphonia globulifera Linnaeus f., (Manil marécage).
Leurs effectifs, accroissement moyen, diamètre moyen, diamètre maximum dans le fichier de données, ainsi que la valeur moyenne de l'indice NBD (très liée à celle du diamètre) pour chacune d'elles figurent dans le tableau VII (voir également figure $6 a$ et $6 b$ ). Dans une classification basée essentiellement sur des critères de croissance et de structure diamétriques, Favrichon [22] a rattaché ces espèces respectivement :

- i) Bocoa prouacensis et Sloanea guianensis au groupe 1 : espèces tolérantes (à l'ombrage) des étages inférieurs ;

- ii) Licania micrantha au groupe 2 : Espèces tolérantes de l'étage supérieur ;

- iii) Dicorynia guianensis et Symphonia globulifera au groupe 3 : espèces semi-tolérantes de l'étage dominant ;

- iv) Carapa procera au groupe 4 : espèces héliophiles de l'étage supérieur.

Tous les diamètres ont été confondus, faute d'effectifs suffisants. Le calcul des coefficients de corrélation linéaire accroissement / indices et leur évolution

Tableau VII. Caractéristiques moyennes, dans le fichier de données (huit parcelles, individus situés à plus de $40 \mathrm{~m}$ des bords), des six espèces retenues. NBD est calculé pour des placettes de rayon $30 \mathrm{~m}$.

\begin{tabular}{lccccc} 
Espèce considérée & Effectif & $\begin{array}{c}\text { Accroissement } \\
\text { moyen annuel } \\
\text { en diamètre } \\
(\mathrm{cm} / \mathrm{an})\end{array}$ & $\begin{array}{c}\text { Diamètre } \\
\text { moyen } \\
(\mathrm{cm})\end{array}$ & $\begin{array}{c}\text { Diamètre } \\
\text { maximum } \\
(\mathrm{cm})\end{array}$ & $\begin{array}{c}\text { Valeur } \\
\text { moyenne } \\
\text { de NBD }\end{array}$ \\
Bocoa prouacensis & 191 & 0,10 & 22,2 & 49,5 & 68,9 \\
Carapa procera & 130 & 0,37 & 22,7 & 61,3 & 61,9 \\
Dicorynia guianensis & 104 & 0,40 & 29,7 & 68,6 & 46,8 \\
Licania micrantha & 316 & 0,20 & 23,2 & 51,2 & 58,3 \\
Sloanea guianensis & 316 & 0,15 & 14,9 & 35,8 & 105,8 \\
Symphonia & 180 & 0,47 & 22,2 & 69,1 & 64,5 \\
globulifera & & & & & \\
- & & & & & \\
\hline
\end{tabular}


(a)

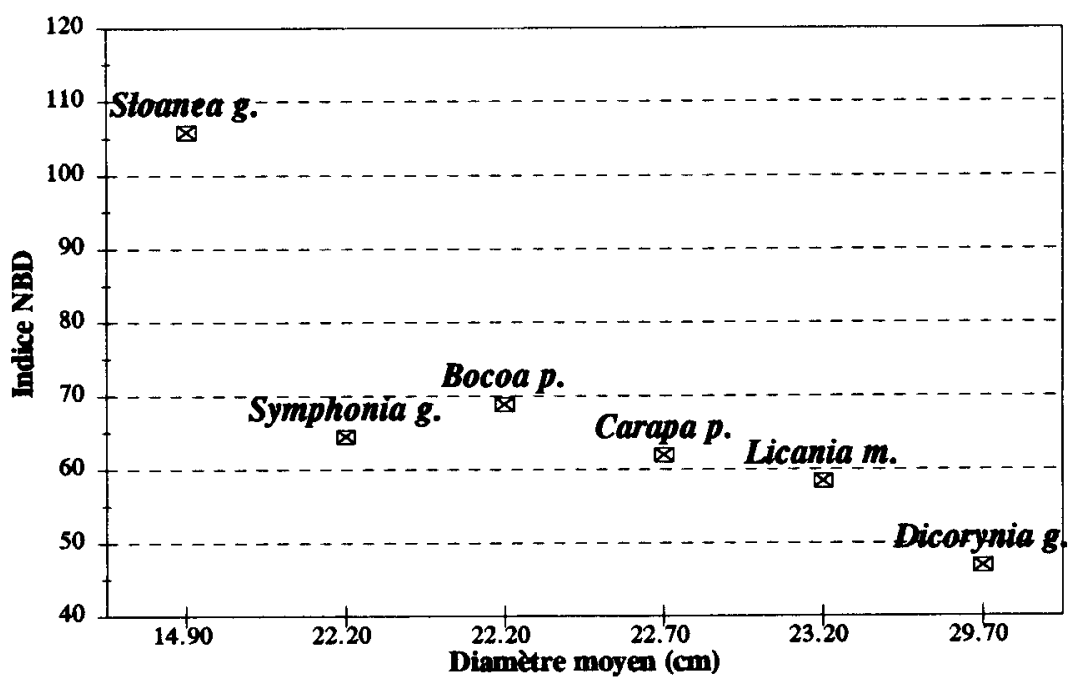

(b)

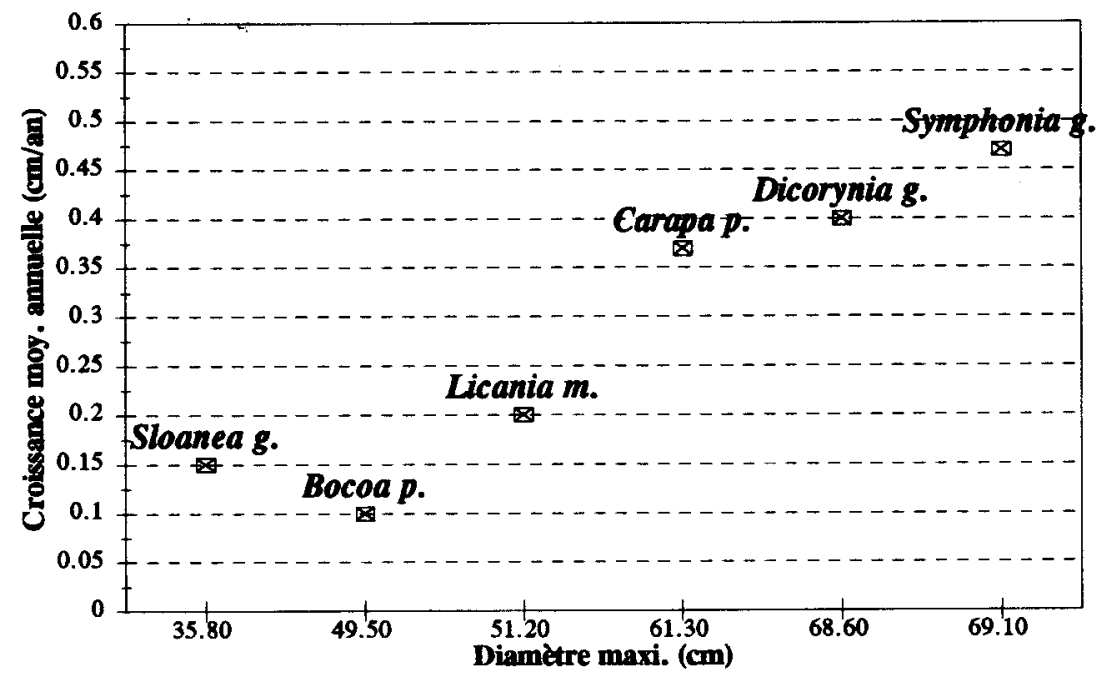

Figure 6. Caractéristiques moyennes des différentes espèces étudiées (pour les individus présents dans le fichier). (a) Représentation de la liaison indice NBD / diamètre (NBD est calculé sur des placettes de rayon $30 \mathrm{~m}$ ). (b) Représentation de la liaison diamètre maximum / accroissement moyen annuel (lissé sur trois ans). 
avec le rayon des placettes font apparaître que :

- i) les indices de compétition les plus liés à l'accroissement dépendent de l'espèce, mais ce sont presque toujours des indices de statut social, et souvent des indices dynamiques (DNBD et DSTD) sauf dans le cas de Dicorynia guianensis, très sensible à NBD ;

- ii) les indices statiques de statut social ne sont pas plus performants chez les espèces exigeantes en lumière que chez les autres. D'une manière générale, toutes les espèces étudiées (sauf Dicorynia guianensis) sont sensibles aux indices statiques bruts NBT et STT qui traduisent une compétition pour l'ensemble des ressources (et pas seulement la lumière) ;

- iii) il n'existe pas de gros écarts de performance entre les indices dynamiques, ce qui n'est pas le cas pour les indices statiques : ainsi, Licania micrantha et Carapa procera, deux espèces ayant pourtant des tempéraments très différents, sont beaucoup moins sensibles aux indices de statut social qu'aux autres, l'inverse se produisant chez Dicorynia guianensis.

-iv ) on notera enfin que chez l'espèce de petite taille Sloanea guianensis, tous les types d'indices, respectivement statiques et dynamiques, ont une efficacité comparable.

Il semble donc que les différentes espèces étudiées soient avant tout sensibles à une compétition exercée par l'ensemble de leurs voisins (prise en compte par les indices statiques NBT et STT), dans un rayon variable dépendant de leurs exigences physiologiques, et à la disparition ou l'apparition dans leur voisinage de sujets les dominant (prises en compte par les indices dynamiques DNBD et DSTD).

Le comportement propre à chaque espèce transparaît moins dans la nature des indices de compétition les plus liés à la croissance que dans leur efficacité. Le tableau VIII (voir aussi le graphique de la figure 7) l'illustre particulièrement bien : on y observe une bonne concordance

Tableau VIII. Meilleurs modèles linéaires à deux variables permettant de décrire l'accroissement moyen annuel en diamètre (sous la forme $\log (\Delta D+c)$ ) des six espèces étudiées.

Espèce considérée

$\begin{array}{cc}\text { Groupe } & \text { Rayon de la } \\ \text { d'espèce } * & \text { placette }\end{array}$

Indices

intervenant

dans le modèle **

$\begin{array}{lllcll}\text { Sloanea guianensis } & 1 & 15 \mathrm{~m} & \Delta N B D, N B D & \mathbf{0 , 2 1} & \mathbf{0 , 2 3} \\ \text { Bocoa prouacensis } & 1 & 30 \mathrm{~m} & \Delta \text { NBD, NBD) } & \mathbf{0 , 2 3} & \mathbf{0 , 2 3} \\ \text { Licania micrantha } & 2 & 25 \mathrm{~m} & \text { NBT, } \Delta \text { STD } & \mathbf{0 , 2 7} & \mathbf{0 , 2 5} \\ \text { Symphonia globuli. } & 3 & 20 \mathrm{~m} & \Delta N B D, N B D & \mathbf{0 , 3 0} & \mathbf{0 , 3 3} \\ \text { Dicorynia guianensis } & 3 & 30 \mathrm{~m} & \text { NBD, }(\Delta N B D) & \mathbf{0 , 3 8} & \mathbf{0 , 3 1} \\ \text { Carapa procera } & 4 & 15 \mathrm{~m} & \Delta N B D, N B D & \mathbf{0 , 4 7} & \mathbf{0 , 3 0}\end{array}$

\footnotetext{
* Selon la classification de Favrichon (1994). Groupe 1: «Espèces tolérantes des étages inférieurs». Groupe 2 : «Espèces tolérantes de l'étage supérieur». Groupe $3:$ «Espèces semi-tolérantes de l'étage dominant». Groupe $4:$ :Espèces héliophiles de l'étage supérieur».

** Les indices entre parenthèse ne sont pas liés linéairement à $\log (\Delta \mathrm{D}+\mathrm{c})$ de manière significative. NB. Les coefficients des indices dans le modèle sont toujours négatifs.
} 
entre degré d'exigence en lumière des espèces et valeur du coefficient de détermination du meilleur modèle linéaire à deux variables permettant de décrire leur accroissement. On notera que les deux indices quasi-systématiquement retenus dans ces modèles sont les mêmes d'une espèce à l'autre. En revanche, la taille des placettes permettant d'obtenir le meilleur résultat varie : de 15 à $30 \mathrm{~m}$ selon les cas. De manière assez logique, des placettes de petite taille paraissent mieux adaptées :

- i) chez les espèces de petite taille (Sloanea guianensis) ;

- ii) chez les espèces héliophiles (Carapa guianensis), sensibles à l'écran venant du voisinage proche ;
- iii) chez les espèces à croissance rapide (Symphonia globulifera) ayant besoin de mobiliser très vite des ressources.

Chez les autres espèces, relativement tolérantes, à croissance plus lente, des placettes de plus grande taille semblent mieux adaptées. Ces conclusions restent évidemment à valider au travers de l'étude d'un plus grand nombre d'espèces ; elles tendent cependant à montrer qu'il existe une marge d'amélioration de la qualité d'un modèle de croissance qui utiliserait des indices simples comme ceux que nous avons étudiés.
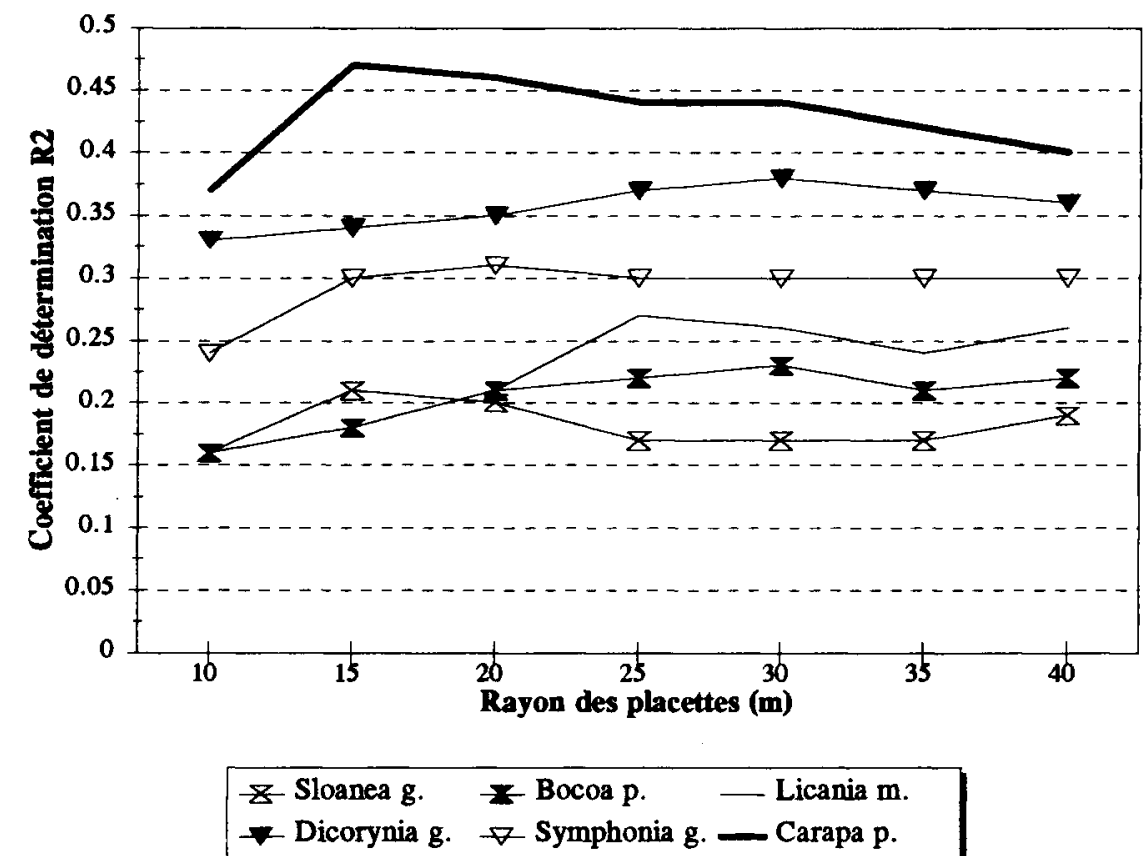

Figure 7. Comparaison, pour différentes espèces, des coefficients de détermination obtenus avec le modèle linéaire $\log ($ accroissement $+0,287)=\mathrm{aIC}_{1}+\mathrm{bIC}_{2}+\mathrm{c} . \mathrm{IC}_{1}$ et $\mathrm{IC}_{2}$ sont les deux indices de compétition qui maximisent les $\mathrm{R}^{2}$ pour chaque espèce. 


\section{CONCLUSION}

Dans le "meilleur des cas », les indices de compétition que nous avons utilisés à Paracou nous ont permis d'expliquer $47 \%$ de la variance observée sur l'accroissement moyen annuel en diamètre d'une espèce (Carapa procera). Toutes espèces confondues cette valeur tombe à $21 \%$. Ces résultats sont conformes à ceux obtenus en forêt dense africaine par Dawkins [16] et Favrichon [21] et peuvent être comparés à ceux que donnent Chai et Lemay [12] pour les forêts marécageuses mixtes du Sarawak (18\% de la variance totale observée sur l'accroissement annuel en diamètre des arbres, toutes espèces confondues).

Si les indices de compétition peuvent donner une idée, certes grossière, de l'accès d'n arbre aux ressources en lumière, eau et éléments minéraux, la disponibilité de ces ressources constitue un facteur non négligeable d'explication de la croissance d'autant que l'on rencontre en forêt dense tropicale des situations très contrastées à grande échelle spatiale : variations rapides (sur quelques mètres) de la profondeur utile des sols, bas-fonds hydromorphes, poches de sable, etc. Mieux approcher cette question, mais aussi celles posées par la nature et l'importance des extensions racinaires, le tempérament des espèces, une meilleure identification des relations sociales susceptibles de s'établir entre deux individus (faire en particulier la part entre relations de compétition et relations de commensalisme), paraissent être des voies à explorer pour améliorer notre compréhension de la croissance d'un arbre dans ce type de forêt.

Compte tenu de ces limites, les deux indices NBD et DNBD (resp. effectif des voisins de taille supérieure à celle du sujet et variation dans le temps de cet effectif) calculés sur des placettes de rayon compris entre 20 et $30 \mathrm{~m}$ nous ont paru être les plus adaptés à une utilisation dans un modèle de croissance. Ils possèdent le gros avantage d'être robustes, simples à calculer et de ce fait aisément utilisables dans un simulateur.

\section{REMERCIEMENTS}

Je tiens à remercier François Houllier pour l'aide qu'il ma apportée dans la réalisation de ce travail, ainsi que Jean Bouchon qui a bien voulu relire et critiquer ce texte.

L'étude a été réalisée dans le cadre d'un DEA et d'une thèse de doctorat financés par le Cirad-Forêt, grâce au travail des nombreuses personnes qui, en Guyane française, alimentent la base de données de Paracou.

\section{ANNEXE - RÉCAPITULATIF DES PRINCIPALES NOTATIONS UTILISÉES DANS LE TEXTE.}

$\mathrm{D}$ : diamètre à $1,30 \mathrm{~m}$, en $\mathrm{cm}$.

$\Delta \mathrm{D}$ : accroissement moyen annuel lissé sur trois ans (cm/an).

DNBD : différence entre les deux valeurs de l'indice NBD, calculées respectivement en 1985 (avant traitement sylvicole) et en 1988 (après traitement sylvicole).

$\mathrm{DNBD}=\mathrm{NBD}_{1988}-\mathrm{NBD}_{1985}$.

DNBT : différence entre les deux valeurs de l'indice NBT, calculées respectivement en 1985 (avant traitement sylvicole) et en 1988 (après traitement sylvicole). DNBT $=\mathrm{NBT}_{1988}-\mathrm{NBT}_{1985}$.

DSTD : différence entre les deux valeurs de l'indice STD, calculées respectivement en 1985 (avant traitement sylvicole) et en 1988 (après traitement sylvicole). DSTD $=$ STD $_{1988}-$ STD $_{1985}$.

DSTT : différence entre les deux valeurs de l'indice STT, calculées respectivement en 1985 (avant traitement sylvi- 
cole) et en 1988 (après traitement sylvicole). DSTT $=$ STT $_{1988}-$ STT $_{1985}$.

IC : indice de compétition quelconque.

NBD : nombre de voisins présents sur une placette circulaire centrée sur l'arbre sujet, et dont le diamètre est supérieur ou égal au sien.

NBT : nombre total de voisins (arbres de plus de $10 \mathrm{~cm}$ de diamètre) présents sur une placette circulaire centrée sur larbre sujet.

$\mathrm{R}$ : rayon d'une placette circulaire centrée sur chaque arbre, utilisée pour identifier ses compétiteurs.

STD : surface terrière cumulée des voisins présents sur une placette circulaire centrée sur l'arbre sujet, et dont le diamètre est supérieur ou égal au sien.

STT : surface terrière cumulée de l'ensemble des voisins (arbres de plus de $10 \mathrm{~cm}$ de diamètre) présents sur une placette circulaire centrée sur l'arbre sujet.

\section{RÉFÉRENCES}

[1] Alder D., Growth and Yield of Mixed Tropical Forests. Consultancy report prepared for the FAO, 1983.

[2] Alemdag I.S., Evaluation of some competition indexes for the prediction of diameter increment in planted White Spruce. Forest Management Institute, Information Report FMR-X-108, 38 p., 1978.

[3] Assmann E., The principles of forest yield study, Pergamon Press, 506 p., 1970.

[4] Atger C., Edelin C., Premières données sur l'architecture comparée des systèmes racinaires et caulinaires, Can. J. Bot. 72 (1994) 963-975.

[5] Bachacou J., Décourt N., Étude de la compétition dans des plantations régulières à l'aide de variogrammes, Ann. Sci. For. 33 (1976) 177-198.

[6] Bella I.E., A new competition model for individual trees, For. Sci. 17 (1971) 364-372.

[7] Besse F., Contribution à l'étude de l'influence de l'environnement sur la croissance du Ba en forêt de Côte-d'Ivoire. DEA de biologie et physiologie végétales, université de Nancy I / CTFT, 48 p., 1984.

[8] Biging G.S., Dobbertin M., A comparison of distance-dependent competition measures for height and basal area growth of individual conifer trees, For. Sci. 38 (1992) 695-720.

19] Bouchon J., Sur l'espace vital des arbres forestiers, Document de la Station de Sylviculture et de Production du CNRF-Inra, 6909, 12 p., 1969.

|10| Bouchon J., Structure des peuplements forestiers, Ann. Sci. For. 36 (3) (1979) 175-209.

[11] Burton P.J., Some limitations inherent to static indices of plant competition, Can. J. For. Res. 23 (1993) 2141-2152.

[12] Chai F.Y.C., Lemay V.M., Development and testing of diameter increment models for mixed swamp forests of Sarawak, For. Ecol. Manage. 58 (1993) 51-64.

[13] CTFT, Spécial Guyane, Bois et Forêts des Tropiques, (1990) p. 219 et 220, 157 et 105.

[14] Daniels R.F., Simple competition indices and their correlation with annual loblolly pine tree growth, For. Sci. 22 (1976) 454-456.

[15] Daniels R.F., Burkhart H.E., Clason T.R., A comparison of competition measures for predicting growth of loblolly pine trees, Can. J. For. Res. 16 (1986) 1230-1237.

[16] Dawkins H.C., The productivity of tropical high-forest trees and their reaction to controllable environment, Balliol College, Nuffield Foundation research fellowship, 1960-1963, Commonwealth Forestry Institute, Oxford, 111 p + tab, 1966.

[17] Dreyfus P., Compétition et croissance dans de jeunes peuplements résineux. Aspects biologiques, écologiques et sylvicoles, thèse d'écologie forestière, université de droit, d'économie et des sciences d'Aix-Marseille, faculté des sciences et techniques de Saint-Jérôme, 1988.

[18] Edelin C., L'architecture monopodiale l'exemple de quelques arbres d'Asie tropicale, thèse, université des sciences et techniques du Languedoc, Académie de Montpellier, 243 p., 1984.

[19] Ek A.R., Dudek A., Development of individual tree based stand growth simulators : progress and applications, Workshop on Forest Succession, Mountain Lake, USA, June 814, 1980, 125, 1980.

[20] Ek A.R., Monserud R.A., Trials with program FOREST: growth and reproduction simulation for mixed species even-or uneven-aged forest stands, in: Fries J. (Ed.), Growth Models for Tree and Stand Simulation, Royal College of Forestry, Stockholm, Res. Notes 30, 56-73, 1974.

[21] Favrichon V., Sur quelques relations entre la croissance des arbres et la structure du peuplement en forêt semi-décidue (République Centrafricaine), mémoire du DEA Ecosystèmes Forestiers Tropicaux, Université Paris VI, 40 p., 1991.

[22] Favrichon V., Classification des espèces arborées en groupes fonctionnels en vue de la réalisation d'un modèle de dynamique de peuplement en forêt guyanaise, Rev. Ecol. (Terre et Vie) 49 (1994) $379-403$.

123] Favrichon V., Modèle matriciel déterministe en temps discret, Application à l'étude de la 
dynamique d'un peuplement forestier tropical humide (Guyane française), thèse, université Claude Bernard, Lyon I / Cirad, 252 p + annexes, 1995.

[24] Gerrard D.J., Competition quotient : an index of the competitive stress affecting individual forest trees, Michigan State University, Department of Forestry, 65 p., 1967.

[25] Glover G.R., Hool J.N., A basal area ratio predictor of loblolly pine plantation mortality, For. Sci. 25 (1979) 275-282.

[26] Gourlet-Fleury S., Indices de compétition : les possibilités d'application à la gestion en forêt dense tropicale humide, mémoire bibliographique du DEA d'analyse et de modélisation des systèmes biologiques, I.A.S.B.S.E., Université Claude Bernard, Lyon I / Cirad, $30 \mathrm{p}+$ annexes, 1992a

[27] Gourlet-Fleury S., Simulation d'éclaircies sur le dispositif sylvicole de Paracou (Guyane française) : recherche de méthodes d'intervention en forêt, après exploitation, Mémoire technique du DEA d'analyse et de modélisation des systèmes biologiques, I.A.S.B.S.E., Université Claude Bernard, Lyon I / Cirad, 34 p + annexes, 1992b.

[28] Gourlet-Fleury S., Montpied P., Dynamique des peuplements denses forestiers en zone tropicale humide : ébauche d'un modèle d'arbre à Paracou (Guyane française), Rev. Ecol. (Terre et Vie) 50 (1995) 283-302.

[29] Hallé F., OIdeman R.A.A., Essai sur l'architecture et la dynamique de croissance des arbres tropicaux, collection de monographies de botanique et de biologie végétale, $\mathrm{n}^{\circ} 6$, Masson, 178 p., 1970.

[30] Hamilton G.J., The dependence of volume increment of individual trees on dominance, crown dimensions, and competition, Forestry 42 (1969) 133-144.

[31] Hatch C.R., Gerrard D.J., Tappeiner J.C., Exposed crown surface area : a mathematical index of individual tree growth potential, Can. J. For. Res. 5 (1975) 224-228.

[32] Hegyi F., A simulation model for managing jack-pine stands, in: Fries J. (Ed.), Growth Models for Tree and Stand Simulation Royal College of Forestry, Stockholm, Res., Notes 30 (1974) 74-87.

[33] Houllier F., Bouchon J., Birot Y., Modélisation de la dynamique des peuplements forestiers : état et perspectives, Rev. For. Fran. 53 (1991) 87-105.

[34] Houllier F., Analyse et modélisation de la dynamique des peuplements forestiers, applications à la gestion des ressources forestières, Mémoire présenté devant l'Université Claude Bernard, Lyon I pour l'obtention de l'habilitation à diriger des recherches, 67 p., 1992.

[35] Jack W.H., Single tree sampling in evenaged plantations for survey and experimentation, Congrès IUFRO, Munich, 379-403, 1967.

[36] Jacquard P., Manifestation et nature des relations sociales chez les végétaux supérieurs, Oecol. Plant. 3 (1968) 137-168.
[37] Johnson E.W., Relationship between point density measurements and subsequent growth of southern pines, Bull. of Auburn University, Agricultural Experiment Station, 447 (1973) 45 p.

[38] Kohyama T., Simulating stationary size distribution of trees in rain forests, Ann. Bot. 68 (1991) 173-180.

[39] Kohyama T., Density-size dynamics of trees simulated by a one-sided competition multispecies model of rain forest stands, Ann. Bot. 70 (1992a) 451-460.

[40] Kohyama T., Size-structured multi-species model of rain forest trees, Funct. Ecol. 6 (1992b) 206-212.

[41] Krajicek J.E., Brinkman K.A., Gingrich S.F., Crown competition, A measure of density, For. Sci. 7 (1961) 35-42.

[42] Latham R.P., Competition estimator for forest trees, Photogrammetric engineering 38 (1972) 48-50.

[43] Lieberman D., Lieberman M., Forest tree growth and dynamics at La Selva, Costa Rica (19691982), J. Trop. Ecol. 3 (1987) 347-358.

[44] Lieberman D., Lieberman M., Hartshorn G., Peralta R., Growth rates and age-size relationships of tropical wet forest trees in Costa Rica, J. Trop. Ecol. 1 (1985) 97-109.

[45] Maitre H.F., Projet de recherches sylvicoles sur les peuplements naturels en forêt dense guyanaise, Rapport interne, CTFT, 1982.

[46] Mitchell K.J., Simulation of the growth of even-aged stands of white-spruce, Bull. Yale University School of Forestry 75 (1969) 48 p.

[47] Mitchell K.J., Dynamics and simulated yield of Douglas-fir, For. Sci., Monograph 17 (1975) $38 \mathrm{p}$.

[48] Moore J.A., Budelsky C.A., Schlesinger R.C., A new index representing individual tree competitive status, Can. J. For. Res. 3 (1973) 495-500.

[49] Nance W.L., Grissom J.E., Smith W.R.,A new competition index based on weighted and constrained area potentially available, in: Forest Growth Modeling and Prediction, 50 Minneapolis, USA, August 24-28, 1987, 134-142.

[50] Oldeman R.A.A., L'architecture de la forêt guyanaise, mémoires Orstom, vol 73 (1974) 198 p + annexes.

[51] Oldeman R.A.A., Van Dijk J., Diagnosis of the temperament of tropical rain forest trees, in: Gomez-Pompa A., Whitmore T.C., Hadley M. (Ed.), Rain Forest Regeneration and Management, Unesco, Man and the Biosphere Series, vol. 6 (1989) 21-63.

[52] Opie J.E., Predictability of individual tree growth using various definitions of competing basal area, For, Sci. 14 (1968) 314-323.

[53] Ottorini J.M., Aspects de la notion de densité et croissance des arbres en peuplement, Ann. Sci. For. 35 (1978) 299-320. 
[54] Prothery N., Recherches sylvicoles sur les peuplements naturels en forêt dense guyanaisc, présentation de quelques résultats densemble 11 ans après la mise en place du dispositif, Rapport interne Cirad-Forêt, 42 p + annexes, 1995.

[55] Pretzsch V.H., Wuchsmodelle für mischbestände als herausforderung für die waldwachstumsforschung, Forstw Cbl II 1 (1992) 87-105.

[56] Schmitt L., Recherches sylvicoles sur les peuplements naturels en forêt dense guyanaise, phase préliminaire : localisation du dispositif principal, Rapport interne, CTFT, 38 p., 1984.

[57] Schmitt L., Recherches sylvicoles sur les peuplements naturels en forêt dense guyanaise, Présentation des résultats issus de la première campagne de mesures (état du dispositif à l'origine), Rapport interne, CTFT, 43 p., 1985.

[581 Schmitt L., Étude des peuplements naturels en forêt dense guyanaise, Compte-rendu de mise en application des traitements sylvicoles sur le dispositif de Paracou, Rapport interne, CTFT, 51 p., 1989.

[59] Smith S.H., Bell J.F., Using competitive stress index to estimate diameter growth for thinned Douglas-fir stands, For. Sci. 29 (1983) 491-499.

[60] Spurr S.H., A measure of point density, For. Sci. 8 (1962) 85-96.

[61] Steneker G.A., Jarvis J.M., A preliminary study to assess competition in a white spruce, trembling aspen stand, For. Chron. 39 (1963) 334-336.
[62] Stone E.L., Kalisz P.J., On the maximum extent of tree roots, For. Ecol. Manage. 46 (1991) 59-102.

[63] Swaine M.D., Lieberman D., Putz F.E., The dynamics of tree populations in tropical forest : a review, J. Trop. Ecol. 3 (1987) 359-366.

[64] Synnott T.J., A manual of permanent plot procedures for tropical rainforests, Department of Forestry, Commonwealth Forestry Institute, University of Oxford, Tropical Forestry Papers $\mathrm{n}^{\circ} 14,67$ p., 1979.

[65] Tomé M., Burkhart H.E., Distance-dependent competition measures for predicting growth of individual trees, For. Sci. 35 (1989) 816-831.

[66] Vanclay J.K., Aggregating tree species to develop diameter increment equations for tropical rainforests, For. Ecol. Manage. 42 (1991) 143-168.

167) Wan Razali B.W.M., Development of a generalized forest growth and yield modelling system for mixed tropical forests of peninsular Malaysia, PhD, University of Washington, 177 p., 1986.

[68] Wadsworth F.H., Parresol B.R., Colon J.C.F., Tree increment indicators in subtropical wet forest, in: Growth and Yield in Tropical Mixed/Moist Forests, Kuala-Lumpur, 20-24 Juin 1988, 15 p., 1988. 